\title{
Machine learning-accelerated quantum mechanics-based atomistic simulations for industrial applications
}

\author{
Tobias Morawietz $^{1}$ D $\cdot$ Nongnuch Artrith $^{2}$ D
}

Received: 14 July 2020 / Accepted: 26 September 2020 / Published online: 9 October 2020

(c) The Author(s) 2020

\begin{abstract}
Atomistic simulations have become an invaluable tool for industrial applications ranging from the optimization of proteinligand interactions for drug discovery to the design of new materials for energy applications. Here we review recent advances in the use of machine learning (ML) methods for accelerated simulations based on a quantum mechanical (QM) description of the system. We show how recent progress in ML methods has dramatically extended the applicability range of conventional QM-based simulations, allowing to calculate industrially relevant properties with enhanced accuracy, at reduced computational cost, and for length and time scales that would have otherwise not been accessible. We illustrate the benefits of ML-accelerated atomistic simulations for industrial R\&D processes by showcasing relevant applications from two very different areas, drug discovery (pharmaceuticals) and energy materials. Writing from the perspective of both a molecular and a materials modeling scientist, this review aims to provide a unified picture of the impact of ML-accelerated atomistic simulations on the pharmaceutical, chemical, and materials industries and gives an outlook on the exciting opportunities that could emerge in the future.
\end{abstract}

Keywords Quantum mechanics · Machine learning $\cdot$ Neural networks $\cdot$ Drug discovery $\cdot$ Energy materials $\cdot$ Industrial applications

\section{Introduction}

Computational methods play an increasingly important role in $\mathrm{R} \& \mathrm{D}$ processes across the pharmaceutical, chemical, and materials industries. Computer-aided drug design [1-3] has the potential to lower the cost, decrease the failure rates, and speed up the discovery process. Computational materials methods help to identify novel materials $[4,5]$, for example, for renewable energy applications [6] such as catalytic energy conversion [7] and energy storage [8]. Results from atomistic simulations aid in the interpretation of experimental measurements and give insights into the structure,

Nongnuch Artrith

nartrith@atomistic.net

Tobias Morawietz

tobias.morawietz@bayer.com

1 Bayer AG, Pharmaceuticals, R\&D, Digital Technologies, Computational Molecular Design, 42096 Wuppertal, Germany

2 Department of Chemical Engineering, Columbia University, New York, NY 10027, USA dynamics and mechanisms of processes occurring on the atomic scale.

In the last decades a new class of atomistic simulation techniques has emerged that combines machine learning (ML) with simulation methods based on quantum mechanical (QM) calculations. Such ML-based acceleration can dramatically increase the computational efficiency of QM-based simulations and enable to reach the large system sizes and long timescales required to access properties with relevance for industry.

Here, we review a selection of ML-accelerated QM methods and their applications to drug design and materials discovery. In the next section we briefly summarize the two main conventional approaches for atomistic simulations, based on molecular mechanics (MM) and QM, respectively, and we show how ML can help overcome their limitations. This is followed by a discussion of recent methodological advances in ML-based interatomic potentials (force fields) for the modeling of complex molecular and materials systems. Finally, we review recent applications of these methods in the fields of drug discovery and materials design. We show that ML-accelerated QM simulations have now 
matured to the point where they can have a large impact on industrial processes.

\section{Atomistic simulation methods}

The central quantity in atomistic simulations is the potentialenergy surface (PES), a high-dimensional function of the position of all atoms in the system. The potential energy is the basic ingredient for Monte Carlo (MD) simulations, while the derivative of the PES yields the atomic forces that are required to numerically solve Newton's equations of motion in molecular dynamics (MD) simulations $[9,10]$. The choice between MD and MC simulations depends on the system and physical process one wants to investigate. MC methods can be employed to obtain structural properties, are efficient in overcoming energy barriers, and can be used for simulating processes in which the number of particles varies. The continuous trajectories generated in MD simulations on the other hand allow to obtain dynamic properties such as vibrational spectra and diffusion coefficients.

When applying atomistic simulations to a given research question, one of the most important considerations is the choice of the simulation method that describes the PES and produces the energy and (possibly) atomic forces that drive the simulation. Depending on the degree of physical approximation, simulation methods are more or less computationally efficient.

Physically most accurate and computationally most expensive are non-empirical QM-based methods that describe the electronic structure and the atomic structures with all degrees of freedom. QM-based simulations are typically limited to small system sizes of less than thousand atoms and short time scales on the order of picoseconds. On the other end of the scale are simulation methods based on empirical molecular mechanics (MM) that do not explicitly describe the electronic structure and may additionally coarse-grain atomic structures by removing select degrees of freedom. Simulation methods that are direct non-empirical approximations to QM are (usually) transferable across the periodic table and across different atomic structures (e.g., organic molecules, bio-polymers, inorganic solids), whereas empirical methods are parametrized for a specific application and are typically not transferable to other situations.

Finally, the usability of a simulation method also depends on the availability of accessible and well-documented software implementations. Hence, the choice of simulation method depends on the physical approximation that is called for by the given research question, and is generally informed by the following four aspects:

1. The types of physical approximations made,

2. The computational efficiency of the method,
3. Its transferability, and

4. Its usability.

Note that a specific research question also determines the relevant length and time scales (e.g., proteins vs. small molecules), and a given application might simultaneously call for high physical accuracy and large length/long time scales. Such research questions cannot be addressed with conventional simulation methods. Novel ML methods, discussed in Sect. Machine learning potentials for atomistic simulations, can overcome this limitation.

A schematic overview of the interrelationship of physical approximation and computational efficiency is shown in Fig. 1. In the following, we briefly review conventional atomistic simulation methods before discussing how these methods can be accelerated and generalized using ML techniques.

\section{MM-based simulations}

In MM-based simulations analytical functions with a small number of parameters often derived from experimental input are employed to describe the PES. They are typically developed for a specific system or application and are called force fields in the context of bio-molecular simulations [11] or interatomic potentials for the description of materials systems [12]. Commonly used force fields (such as AMBER [13], CHARMM [14], GROMOS [15], and OPLS [16]) are computationally very efficient since they employ simple pairwise interaction terms and fixed atomic charges. They also rely on the definition of atomic connectivities and atom types and are therefore non-reactive. Examples for interatomic potentials for the descriptions of solids and surfaces are the Lennard-Jones pair potential [17], the embedded atom model (EAM) [18], and bond order potentials such as the Tersoff potential [19].

MD simulations with force fields have become a key technique for different stages in the drug discovery pipeline [20, 21]. One specific example is their use in the early discovery phase for the calculation of relative binding affinities of ligand molecules to a protein binding site. The ability to efficiently calculate the associated binding free energy [22-24] can provide valuable contributions to the ligand optimization phase, allowing to rank ligands, optimize selectivity, and estimate off-target interactions. With recent advances in the free energy methods and underlying simulation models, the calculation of free energies from atomistic MD simulations has become a reliable tool with several examples of successful industrial applications [25-29]. Multi-scale approaches in which atomistic force fields are combined with coarse-grained models that were built for specific applications can further reduce the computational cost and allow 


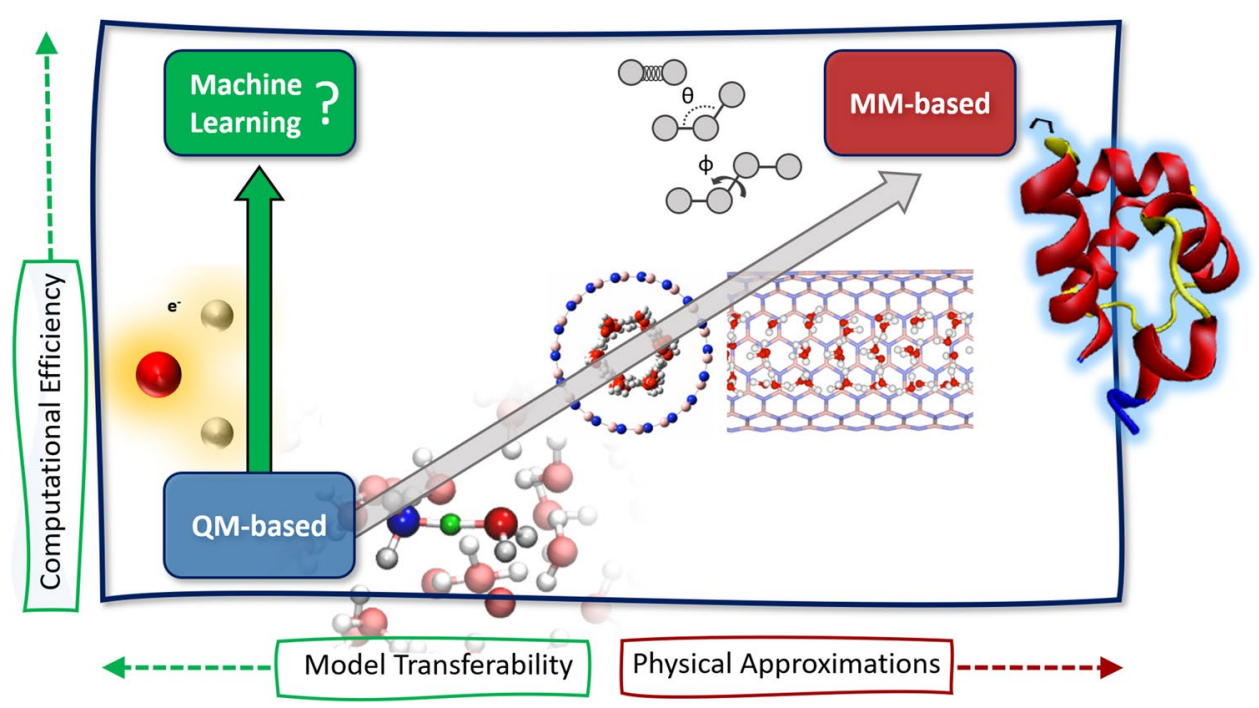

Fig. 1 Atomistic simulation methods can be broadly categorized into two classes depending on the way the system is described: using quantum mechanical (QM) calculations based on the electronic structure or molecular mechanics (MM) methods with predefined functional forms. Due to their higher computational cost QM-based simulations are limited to smaller systems while MM-based methods are more efficient but rely on many approximations and are often derived from experimental input. The goal of QM-based machine learning is to raise the efficiency of QM methods without sacrificing their transferability, predictive power and ability to describe complex bonding patterns including the breaking and forming of chemical bonds

\section{QM-based simulations}

channels [30].

A general drawback of empirical MM simulations is the fact that the obtained results depend on the experimental data the models are based on, which restricts their predictive power and transferability to conditions not included in the optimization process. Standard force fields for example are parametrized to a limited set of chemical elements and cannot be easily applied to metal-containing proteins. Another serious limitation is the inability to describe the breaking and forming of chemical bonds, prohibiting their use for industrially relevant processes such as the investigation of enzymatic reactions for covalent inhibitor design [31] or of catalytic reactions at metal oxide surfaces [32].

To address these limitations several extensions of empirical force fields and potentials have been developed. Examples are approaches that go beyond atom types using the SMARTS chemical perception language [33] developed by the Open Force Field Initiative [34], reactive force fields that allow the breaking of chemical bonds [35], and the development of frameworks for systematic and reproducible parametrization procedures [36, 37]. All these approaches have in common that the employed functional form to approximate the PES is predetermined and for the sake of efficiency approximated by simplified functions with a small number of model parameters.
QM-based simulation methods (also called ab initio molecular dynamics [AIMD] or first principles simulations) [38, 39] circumvent the problem of defining a functional form for the PES. Here, energy and atomic forces are obtained on-the-fly, in each step of the simulation, by (approximately) solving the Schrödinger equation using an electronic structure method like density-functional theory (DFT) [40]. QM simulations are fully reactive and can describe the complex bonding patterns, polarization effects and charge transfer processes that govern the behaviour of biological systems [41]. In combination with path integral approaches also nuclear quantum effects (NQEs) [42, 43] like zero-point motion and tunneling can be included, processes that are important for the description of systems containing hydrogen-bond networks and acidic protons. QM-based simulations can be applied to obtain a large set of materials properties, for example the stability of crystal structures, elastic constants, and transport phenomena. In addition to energies and forces, other observables can be directly calculated by QM methods such as dipole moments, polarizabilities, chemical shifts, and phonon frequencies for the spectroscopic characterization of molecular and materials systems.

While MM-based simulations are only possible when reliable force fields (or interatomic potentials) for the given system are available, QM methods are in principle applicable to all chemical species. In practice, there is no single QM method that is computationally affordable and reliable 
for every system, and the approximations made in a chosen method still need to be carefully validated [40]. The largest bottleneck of QM-based simulations is the high computational cost of the electronic structure calculations that have to be executed in each simulation step. Even for efficient QM methods such as DFT the algorithmic scaling is typically of order $\mathcal{O}\left(N^{3}\right)$ in the number of electrons $N$, which means that an increase in system size by a factor of 10 leads to an increase in processing time of a factor of 1000 . This severely limits the application of QM simulations to small, often idealized model structures containing not more than a few hundred atoms. Semi-empirical methods [44-47] such as density functional tight binding (DFTB) [48-50] lower the computational burden and can even describe full proteins [51] but their efficiency comes at the cost of transferability and accuracy.

\section{Overcoming the limitations of QM-based simulations with machine learning}

As detailed in the previous two sections, MM-based atomistic simulation methods can be computationally highly efficient but have limited transferability owing to their high degree of physical approximation. Conversely, QM-based methods can be highly accurate but are computationally too demanding for many applications of industrial relevance. While mixed quantum mechanics/molecular mechanics (QM/MM) approaches [52-55] can, in principle, combine the strengths of both worlds, QM/MM is technically involved and usability is therefore not always given. Modifications [56, 57] of the original Car-Parrinello method [38] can reduce the computational burden of QM simulations to some extend but are still much more costly compared to MM-based simulations. If a research question requires simultaneously high accuracy and high computational efficiency (the top left corner of the schematic in Fig. 1), this means in practice often that it cannot be addressed with conventional atomistic simulation methods.

To overcome this limitation, a number of methodologies based on ML have been developed during the last decades. The purpose of the different ML strategies generally falls into one of the following four categories:

1. Extension of the applicability range of QM simulations to larger length and time scales;

2. Prediction of properties calculated from QM methods;

3. Automated analysis of simulation data; and

4. Inversion of atomistic calculations to generate atomic structures for a given set of properties.

Strategy (1) is based on the development of machine-learning potentials (MLP) that achieve an accuracy that is close to (or identical to) QM-based methods but at significantly reduced computational cost that scales linearly with the system size. MLPs can be taken as drop-in replacement for conventional interatomic potentials or force fields, which ensures a high usability.

In strategy (2), ML models are trained to yield the outcome of QM-based calculations either using optimized structures or configurations obtained from atomistic simulations. Examples are ML predictions of atomization energies $[58,59]$ of small organic molecules, nuclear magnetic resonance (NMR) shifts [60] and band gaps [61-63] of inorganic solids, and adsorption energies of electrocatalysts [64]. By design, ML models of type (2) are less general than MLPs as they are specific to one or few QM properties and do not easily transfer to others. The increasing availability of QM databases enables training such ML models for an ever growing number of QM properties, and we discuss examples in Sect. Spectroscopic techniques for structure characterization.

MD and MC simulations of complex atomistic systems can yield data that are challenging or time-consuming to interpret for humans, such as MD trajectories with frames (atomic coordinates) from billions of time steps. Strategy (3) uses ML techniques for the analysis of simulation data, for example for the automatic identification of crystal structures [65] or the extraction of free energy surfaces from enhanced-sampling MD simulations [66, 67].

Finally, the inverse design strategy (4) holds great promise for the future of molecular and materials design but is currently in its infancy with few published examples. For examples of inverse molecular design, we refer to a recent review by Sanchez-Lengelin and Aspuru-Guzik [68]. In general, methods that implement ML models of type (4) are not yet standardized and usability is therefore generally not yet given.

In this review we focus on ML approaches of strategy (1) and (2), i.e. MLPs for accelerated simulations and ML models that predict the outcome of QM calculations, since those are the most mature and offer a reasonable balance of usability and pay-off for industrial applications. More general applications of ML approaches, for example for retrosynthesis $[69,70]$, direct prediction of experimental properties [71-73], and molecule generation and optimization [74-76], are discussed in references [77-80]. In the following section, we discuss different types of MLPs and approaches for their construction.

\section{Machine learning potentials for atomistic simulations}

As discussed in the previous section, atomistic simulations sample the PES of collections of atoms, and the description of the PES may be either based on first principles QM or on 
approximate physical or ad-hoc mathematical expressions. If a PES is described by a mathematical function that does not have any direct correspondence in the laws of physics, the PES can nevertheless be highly accurate if it interpolates the true QM potential energy faithfully for all relevant atomic arrangements. For example, the mathematical form of the repulsive branch of the (12-6) Lennard-Jones pair potential [17] was originally chosen for computational simplicity and does not reflect the true exponential behavior known from QM, but the Lennard-Jones potential nevertheless describes noble gas dimers with great accuracy because it provides a good interpolation of the QM potential energy for all relevant bond lengths. An atomistic simulation of an argon dimer with a Lennard-Jones potential can therefore be just as accurate as a full quantum-mechanical calculation, while it is computationally more efficient by several orders of magnitude.

What if we had a flexible mathematical function that is able to accurately interpolate the QM potential energy for any arbitrary atomic system, not only for dimers or select classes of materials?

As it turns out, it can be shown that artificial neural networks (ANN) [81] with finite numbers of parameters can represent any real-valued continuous function, such as PESs, with arbitrary accuracy. This is in simple terms the conclusion of the universal approximation theorem [82, 83], and similar theorems have been derived also for other ML regression methods such as Gaussian process regression (GPR) [84]. Hence, carefully constructed ML regression models can in principle replace any QM PES without loss of accuracy.

The regression or interpolation of PESs with ML, is at the core of $M L$ potentials. Fig. 2 shows an overview of the main steps involved in the construction and application of ML potentials for accelerated QM-based simulations:
(1) reference calculations, (2) model training, (3) model application. The various ML potential methods differ in the ML method used for regression and the descriptor approach used for the translation of atomic structures to features that are suitable as input for ML models.

Several ML methods have been used for the task of learning PESs, from ANNs [85, 86], to GPR [87, 88], and kernel ridge regression (KRR) [89]. The discussion here focuses on ANN-based ML potentials, which have been applied to the widest range of materials and compositions.

\section{Representation of PESs with ANNs}

On a fundamental level, ANNs are non-linear vector functions that take a vector as input and produce another vector as output. The functional form of ANNs consists of a combination of elemental building blocks that may be interpreted as artificial neurons, since they perform an operation that is on a basic level similar to that of a biological neuron. Each artificial neuron takes the weighted sum of one or more input values $x_{i}$ and applies a non-linear activation function $f_{\mathrm{a}}$ to the result

$y=f_{\mathrm{a}}\left(\sum_{i} a_{i} x_{i}+b\right)$

where $a_{i}$ is the weight of the $i$-th input and $b$ is a bias weight that allows for an additional constant shift that does not depend on the input values. An ANN is the combination of interconnected artificial neurons such that the outputs of some neurons are the inputs of others. In a feed-forward $A N N$, the neurons are organized in layers, and all connections are in one direction, i.e., outputs from all neurons of one layer are the inputs of the neuron of the subsequent

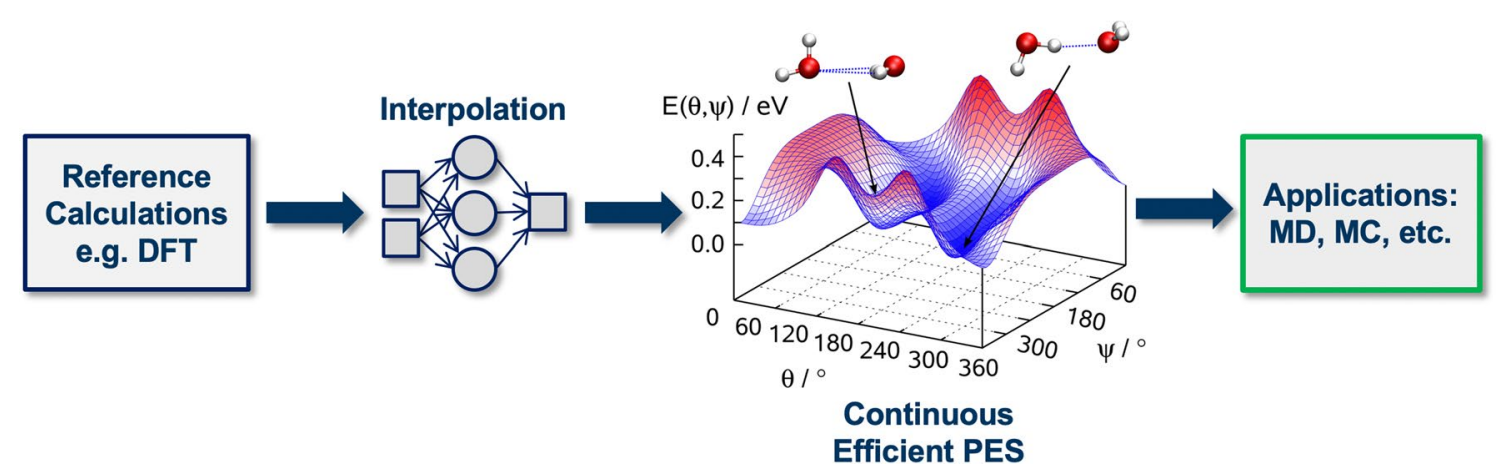

Fig. 2 Workflow for machine learning-accelerated atomistic simulations: first, reference calculations are performed for a set of configurations using a quantum mechanical (QM) method such as densityfunctional theory (DFT). The resulting QM energies (and potentially forces) are then used to train a machine learning model that maps the atomic structure to its corresponding energy and by that learns the potential-energy surface (PES) of the atomistic system. Once trained, the resulting ML model yields a continuous representation of the PES that can be efficiently evaluated and allows to perform molecular dynamics (MD) or Monte Carlo (MD) simulations for larger systems and on longer time-scales than possible with direct QM-based simulations 
layer. The graph representation of a feed-forward ANN is shown under the label "interpolation" in Fig. 2. ANN training is the process of optimizing the weight parameters $\left\{a_{i}\right\}$ and $\{b\}$ for each neuron to reproduce reference data within a training set.

In principle, feed-forward ANNs can be directly used for the interpolation of PESs in the sense that is indicated in Fig. 2. In this scheme, the atomic positions, e.g., the Cartesian coordinates of all atoms, are the input of the ANN, and the potential energy is the output. Variations of this approach have been used in theoretical chemistry since the 1990s [90-93] to accelerate the modeling of select molecular systems.

This naïve interpolation, however, disregards fundamental symmetries of the potential energy with respect to rotation/translation of the atomic structure and the exchange of equivalent atoms. Hence, care must be taken that the ANN-interpolated PES does not exhibit unphysical features. In addition, the input dimension of the ANN is fixed to the number of degrees of freedom of a specific atomistic system, and it is not possible to use the same ANN to predict the potential energy of atomic structures with fewer or more atoms.

Lorenz et al. [94] introduced a transformation of the Cartesian atomic coordinates into a set of coordinates that incorporates the symmetries of the PES before the ANN interpolation, to describe the dissociation of an $\mathrm{H}_{2}$ molecule over the $\operatorname{Pd}(100)$ surface. The limitation to a fixed number of atoms was removed by the high-dimensional neural network potential approach.

\section{High-dimensional neural network potentials}

To overcome the limitations of ANN-interpolated PESs, in 2007 Behler and Parrinello proposed an ANN potential methodology [85] that is based on an implicit decomposition of the total potential energy $E(\sigma)$ of an atomic structure $\sigma$ into atomic energy contributions $E_{i}$

$E(\sigma) \approx \sum_{i}^{\text {atoms }} E_{i}\left(\sigma_{i}\right)$ with $\sigma_{i}=\left\{\mathbf{R}_{j}, t_{j}\right.$ for $\left.\left|\mathbf{R}_{j}-\mathbf{R}_{i}\right| \leq R_{\text {cut }}\right\}$.

In equation (2), $\sigma_{i}$ is the local structural environment of atom $i$ that contains only the coordinates $\mathbf{R}_{\mathbf{j}}$ and chemical species $t_{j}$ of those atoms that are within a cutoff distance $R_{\text {cut }}$ from the position $\mathbf{R}_{i}$ of atom $i$. In the high-dimensional neural network potential method, ANNs are trained to predict the atomic energy $E_{i}$.

Following the idea by Lorenz et al. [94], the ANN input is obtained by representing features of the local atomic environment $\sigma_{i}$ with symmetry functions $[85,95]$ that incorporate the rotational symmetry and the symmetry with respect to the exchange of equivalent atoms. Other symmetry-invariant descriptors or fingerprints of local atomic environments have since been developed, and key methods are reviewed in Sect. Descriptor of the local atomic environment.

The original Behler-Parrinello method was limited to a single atomic species. In 2011, the method was extended to multicomponent compositions by Artrith, Morawietz, and Behler by training separate ANNs for different atomic types $t$ [96]. The total energy in the multicomponent ANN potential method is given by

$E(\sigma) \approx E_{\mathrm{ANN}}(\sigma)=\sum_{t}^{\substack{\text { atom atoms of } \\ \text { types }}} \sum_{i}^{\text {type } \mathrm{t}} \operatorname{ANN}_{t}\left(\widetilde{\sigma}_{i}\right)$

where $\widetilde{\sigma}_{i}$ is the symmetry-invariant descriptor (fingerprint) of the local atomic environment $\sigma_{i}$ and $\mathrm{ANN}_{t}$ is the atomic ANN for atoms of type $t$.

Figure 3 shows a graph representation of a high-dimensional neural network potential including the translation of the atomic coordinates $\mathbf{R}_{i}$ to an invariant fingerprint of the local atomic environment $\widetilde{\sigma}_{i}$ and the prediction of the atomic energies $E_{i}$ by the atomic ANNs.

Note that training ANN potentials that are based on atomic energy contributions is technically more involved than the direct ANN interpolation of the PESs discussed in Sect. Representation of PESs with ANNs, since the atomic energies are not uniquely defined in QM simulation methods and are therefore not directly available as reference. In most ANN potential methods the atomic energy is learned implicitly from the total energy, i.e., the reference data for the ANN potential training are total energies (and

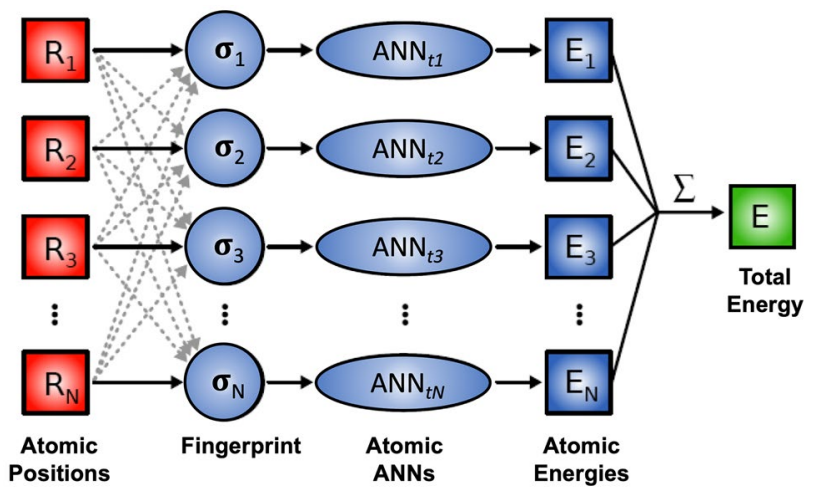

Fig. 3 Diagram of the high-dimensional neural network that combines the atomic ANNs of all atoms in a structure for an $\mathrm{N}$-atom system. The output is the total energy $E$, which is the sum of the individual atomic energy contributions $E_{i}$, which are in turn the outputs of atomic feed-forward ANNs 
its derivatives). Alternatively, the QM total energies can be first decomposed into atomic energy contributions via nonunique schemes [97], which can improve the training efficiency but introduces an additional step in data preparation.

A fundamental assumption of the multicomponent ANN potential approach as expressed in Eq. (3) is that the total energy is entirely given by a sum of short-ranged atomic energy contributions. However, some contributions to the total energy are known to be long-ranged. Specifically, atomic structures with ionic species or ionic bonding contributions exhibit long-ranged electrostatic interactions. Also, long-ranged dispersive (London or van der Waals) interactions are of crucial importance, for example, for (bio)polymers and for adsorption phenomena.

\section{ANN potentials with long-ranged electrostatic interactions}

The energy contribution from the electrostatic interaction of two charged atoms $i$ and $j$ is given by Coulomb's law

$E_{i, j}^{\mathrm{elec}}\left(\mathbf{R}_{i}, \mathbf{R}_{\mathbf{j}}, q_{i}, q_{j}\right)=\frac{1}{4 \pi \varepsilon_{0}} \frac{q_{i} q_{j}}{R_{i j}}$,

where $q_{i}$ and $q_{j}$ are the atomic charges, $R_{i j}=\left|\mathbf{R}_{j}-\mathbf{R}_{i}\right|$ is the interatomic distance, and $\varepsilon_{0}$ is the permittivity of the vacuum. Since Coulomb interactions decay only as $1 / R_{i j}$ with the interatomic distance, they cannot be generally truncated at any cutoff, hence, the ANN potential expression of Eq. (3) would be inappropriate irrespective of the cutoff chosen for the local atomic environment. Note that electrostatic interactions in dense media, such as solids or liquids, are screened and can often be treated as effectively short-ranged [98]. It should be also kept in mind that the distance dependence of the ANN forces is twice as large as the chosen cutoff that defines the size of the local atomic environments [96, 99-101]. If screening cannot be assumed, the ANN potential approach needs to be extended.

To address this need, Artrith, Morawietz, and Behler proposed an extension of the ANN potential method by a second set of ANNs that are trained to reproduce environmentdependent atomic charges [96, 101-103]. In this approach, the total energy is given by

$E_{\mathrm{ANN}}^{\mathrm{total}}=E_{\mathrm{ANN}}^{\mathrm{short}}+E_{\mathrm{ANN}}^{\mathrm{long}}$,

where the short-range energy contribution $E_{\mathrm{ANN}}^{\text {short }}$ is given by the expression of Eq. (3). The long-range energy contribution takes the usual Coulomb form

$E_{\mathrm{ANN}}^{\text {long }}=\sum_{i, j}^{\text {atoms }} E_{i, j}^{\mathrm{elec}}\left(\mathbf{R}_{i}, \mathbf{R}_{\mathbf{j}}, q_{i}, q_{j}\right)$ which can be evaluated, for example, with the Ewald summation technique [104] or using approximate damped techniques such as the pairwise approach by Fennell and Gezelter [105]. The atomic charges in Eq. (6), $q_{k}=\operatorname{ANN}_{t}^{q}\left(\widetilde{\sigma}_{k}\right)$, are represented by ANNs as function of the local atomic environment. A schematic of the electrostatic extension of the high-dimensional ANN potential method is shown in Fig. 4.

The original approach $[96,102]$ trained the charge ANNs on Hirshfeld charges [106]. Since the decomposition of the total charge density into atomic contributions is not uniquely defined, other charge partitioning schemes [107] would have been equally valid. To avoid training potentially illdefined atomic charges directly, Yao et al. trained atomic charges implicitly such that they reproduce molecular dipole moments [108], which are physical observables. In the case of ionic crystals, a static charge approach in which the atomic charges are independent of the environment has also been demonstrated to work [109]. Finally, the restriction of long-ranged electrostatic interactions to the Coulomb form and to explicit atomic charges might be avoidable by introducing an energy term that depends on long-ranged features of the atomic structure, which Grisafi and Ceriotti recently demonstrated for a simplified model [110].

\section{ANN potentials with dispersive interactions}

In addition to splitting off electrostatic interactions, also dispersive van der Waals ( $\mathrm{vdW}$ ) interactions can be treated separately. Morawietz and Behler [111] introduced an extended energy expression,

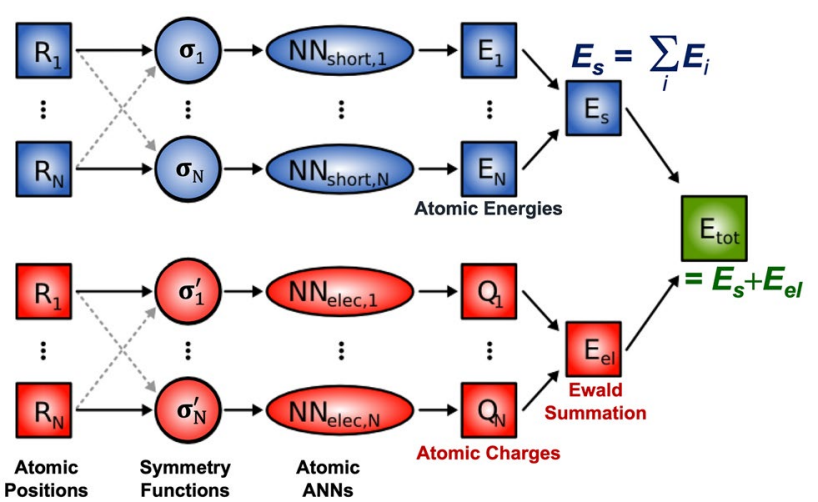

Fig. 4 Diagram of the high-dimensional neural network potential for multicomponent systems: The total energy of the system is obtained as a sum of a short-range energy $\left(\mathrm{E}_{\mathrm{s}}\right)$ obtained as shown in Fig. 3 and a long-range electrostatic energy $\left(\mathrm{E}_{\mathrm{el}}\right)$, which is calculated from atomic charges $Q_{i}$. Both the short-range atomic energies and the atomic charges depend on the local atomic environments and are constructed by atomic ANNs [96] 
$E^{\text {total }}=E_{\mathrm{ANN}}^{\text {short }}+E_{\mathrm{ANN}}^{\mathrm{elec}}+E^{\text {disp }}$,

where $E^{\text {disp }}$ is an analytic correction term which improves the description of dispersion interactions using Grimme's D3 method [112]. A similar approach was taken by Yao et al. [108] based on the D2 correction scheme [113].

While the main reason for introducing explicit electrostatics is their long-ranged nature which cannot be represented by short-range atomic energies, the need to include a separate dispersion term depends on the employed reference method and the system. A vdW correction term might be required if DFT is used as the reference method, since (semi-)local density-functionals suffer from an inaccurate description of these interactions. Having an explicit vdW correction term that is added to the short-range energy represented by the ANN has the additional benefit that its interactions are not truncated at a short distance. However, for the description of homogeneous systems in which longrange forces are screened, it is also valid to add the vdW term to the short-range reference data and train ANNs on
The choice of the optimal descriptor method depends on the application, as some methods are better suited for isolated molecular systems where others were designed for periodic solids. Another factor in the descriptor selection is the balance of computational efficiency and accuracy. Various approaches have been proposed [88, 95, 118-126], and we limit the discussion here to the ones that are most commonly used and are available in public software implementations.

The descriptor introduced with the original method by Behler and Parrinello is based on an representation of the coordinates within the local atomic environments in symmetry functions $[85,95]$. The symmetry functions and modified variants are commonly used as descriptors in public ANN potential implementations, such as ænet [127], AMP [128], ANI [129], TensorMol [108], and N2P2 [130].

Behler proposed two classes of symmetry functions [95], radial functions that capture the interatomic distances within the local atomic environment and angular functions that describe the bond-angle distribution. The symmetry functions have the general functional form

$G_{\text {radial }}\left(\sigma_{i}\right)=\sum_{j \in \sigma_{i}} g_{1}\left(R_{i j}\right) f_{\mathrm{c}}\left(R_{i j}\right), G_{\text {angular }}\left(\sigma_{i}\right)=\sum_{j, k \in \sigma_{i}} g_{2}\left(R_{i j}, R_{i k}, R_{j k}\right) f_{\mathrm{c}}\left(R_{i j}\right) f_{\mathrm{c}}\left(R_{i k}\right) f_{\mathrm{c}}\left(R_{j k}\right)$,

the joined energies and forces. This was for example done to study the impact of $\mathrm{vdW}$ interactions on the properties of ice and liquid water by training ANNs to represent two density-functionals with and without inclusion of a vdW correction term [114].

\section{Descriptor of the local atomic environment}

The explicit or implicit decomposition of the total structural energy, either into atomic contributions as in Eq. (2) or into the contributions of bonds or other fragments is a general feature of transferable ANN potentials. However, the various ANN potential methods developed today differ often in the symmetry-invariant descriptor (fingerprint) used for the feature extraction from atomic or fragment environments.

Widely adopted descriptor methods are based on the expansion of the atomic positions or bond-length and angle distributions. Recently, Xie and Grossman proposed a graph convolution approach as descriptor for molecular and periodic atomic structures [115]. This descriptor was further adapted by Chen et al. [116], who applied it to the development of accurate ML models for property prediction. While most ANN potential methods rely on hand-crafted descriptors that were designed based on chemical intuition, the recent deep ANN potential method by Schütt et al. [117] avoids the need for empirical feature extraction by means of a general convolution approach akin to those used in computer vision. where $f_{\mathrm{c}}$ is a cutoff function that smoothly goes to zero at the cutoff of the local atomic environment, and $g_{i}$ are parametrized functions designed to sample the distributions of bond lengths and angles, respectively. The dependence on the interatomic distance $R_{j k}$ may be omitted in $G_{\text {angular }}$. During the construction of an ANN potential, the number of symmetry functions and the parametrization of the functions $g_{1}$ and $g_{2}$ are meta parameters that have to be optimized. In addition to Behler's original set of symmetry functions [85, 95], the ANN potential implementation ANI introduced a set of modified symmetry functions with slightly different definitions of the functions $g_{i}$ [129]. A schematic of the bond length and angle distribution within a local atomic environment is shown in Fig. 5.

The manual parametrization of $g_{1}$ and $g_{2}$ in the symmetry functions of Eq. (8) has advantages for ordered structures and molecular systems with well-known bonds and angles, but it complicates the construction of general ANN potentials when no such assumptions can be made. Recently, Li et al. proposed a formalism for the automatic optimization of symmetry function parameters based on pair-distribution functions [133].

As an alternative, Artrith, Urban, and Ceder developed a descriptor that is based on a formal expansions of the radial and angular distribution functions in an orthonormal basis set $\left\{\phi_{\alpha}\right\}$ [132]. For the radial distribution function (RDF) around atom $i$, this expansion can be written as 
Fig. 5 Schematic of radial and angular descriptors used for the representation of local atomic environments (left). The descriptor functions extract features that are used as input values for atomic energy ANNs. Separate ANNs for each atomic species (chemical element) are trained, so that the total energy of a binary material consist of two terms (right)

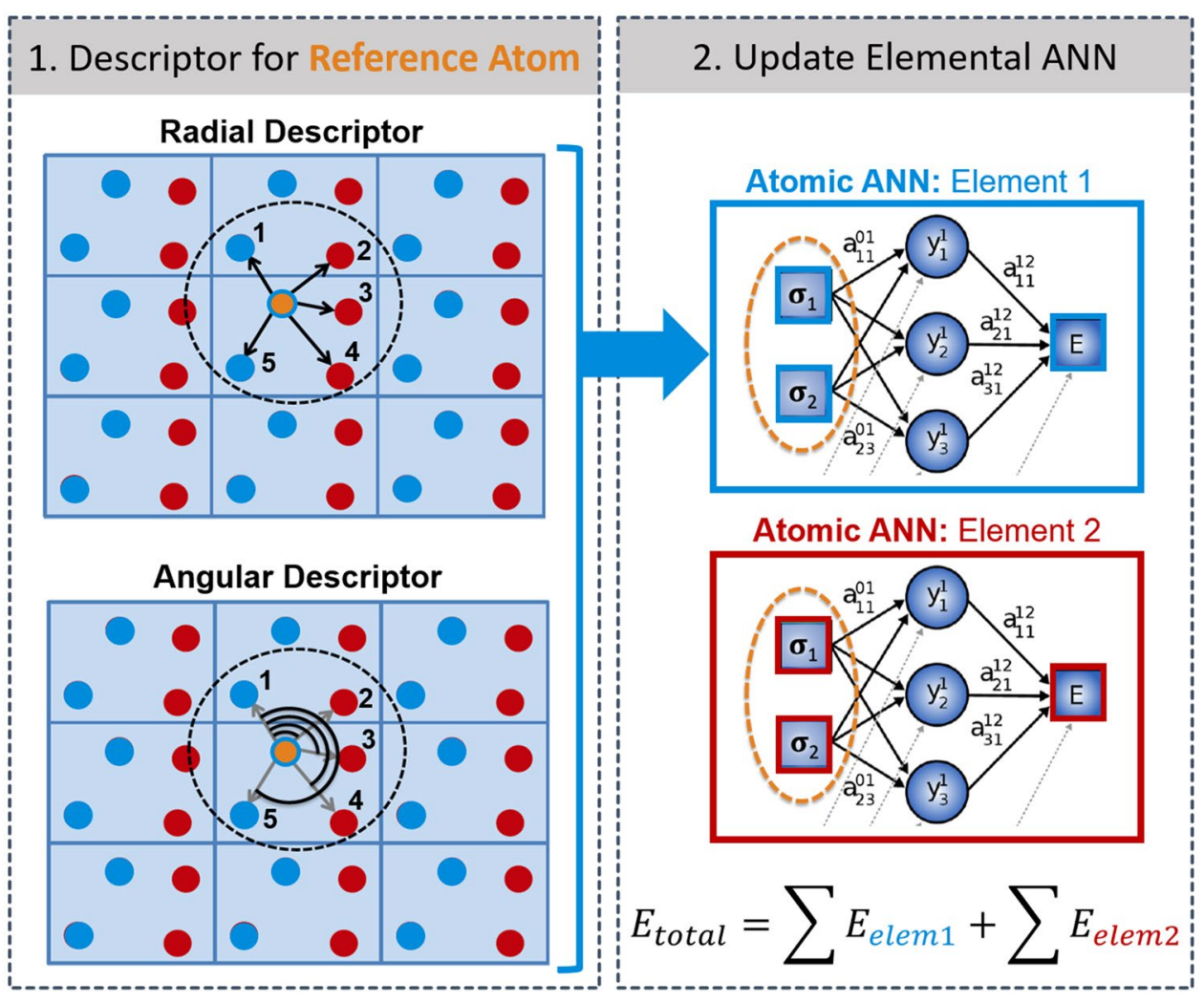

$\operatorname{RDF}_{i}(r)=\sum_{\alpha} c_{\alpha}^{(2)} \phi_{\alpha}(r)$

where the expansion coefficients $\left\{c_{\alpha}^{(2)}\right\}$ are invariant features of the local atomic environment and are given by

$c_{\alpha}^{(2)}=\sum_{\mathbf{R}_{j} \in \sigma_{i}} \phi_{\alpha}\left(R_{i j}\right) f_{\mathrm{c}}\left(R_{i j}\right) w_{t_{j}}$.
The expansion of the angular distribution functions is completely analogous. Equations (9) and (10) introduce the basis functions $\phi_{\alpha}$ and an atom-type (chemical species) specific weight parameter $w_{t_{i}}$. Artrith et al. [132] chose Chebyshev polynomials as orthonormal basis set (see Fig. 6a), and the radial and angular distributions can be refined to arbitrary accuracy by including polynomials with increasing order
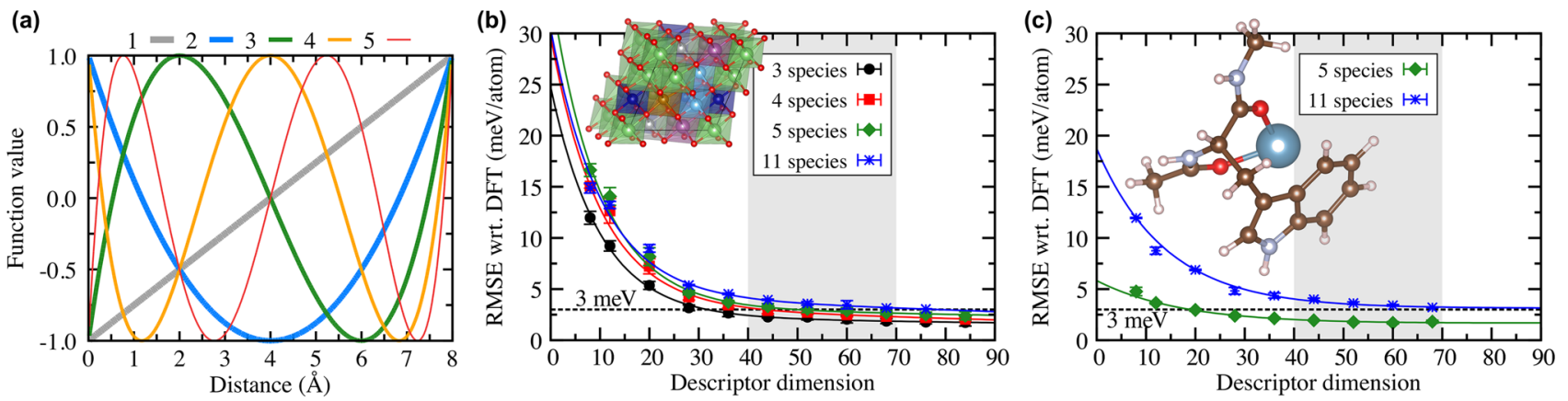

Fig. 6 The Chebyshev descriptor (implemented in ænet [127]) enables the simulation of multicomponent compositions with many different chemical species. (a) Basis functions $\left\{\phi_{\alpha}\right\}$ of Eqs. (9) and (10) (Chebyshev polynomials) up to order 5 for a cutoff radius of $8.0 \AA$. The polynomial of order 0 is constant 1 and not shown. (b) and (c) show the accuracy of artificial neural network (ANN) potentials in terms of the root-mean-squared error (RMSE) compared to the QM reference method (DFT) as function of the size of the structural fin- gerprint (descriptor) for (b) an inorganic solid $\left(\mathrm{Li} M \mathrm{O}_{2}\right)$ with increasing number of chemical species (from the set $\mathrm{Li}, \mathrm{O}, \mathrm{Ti}, \mathrm{Ni}, \mathrm{Mn}, \mathrm{Sc}$, $\mathrm{V}, \mathrm{Cr}, \mathrm{Fe}, \mathrm{Co}$, and $\mathrm{Cu}$ ) and (c) a data set with conformations of the 20 proteinogenic amino acids (5 chemical species: $\mathrm{H}, \mathrm{C}, \mathrm{N}, \mathrm{O}, \mathrm{S}$; green diamonds) and their complexes with divalent cations (amino acid data taken from Ref. [131]). (Reproduced with permission from Ref. [132]. Copyright (2017), American Physical Society.) 
without the need for manual parametrization. Faber et al. [121] previously employed a Fourier expansion.

The symmetry functions allow constructing a representation of the local structure but do not encode the chemical species, which is also needed for an accurate ANN potential. In the original multicomponent ANN potential method [96], the chemical species are distinguished with separate sets of symmetry functions for each combination of two (radial functions) and three (angular functions) chemical species. This approach results in an increase of the descriptor dimension and thus the computational effort with the number of atomic species, which makes it challenging to construct ANN potentials for more than a few chemical elements [86].

The Chebyshev descriptor [132] removes this scaling by introducing a weight parameter, $w_{t_{j}}$ in Eq. (10), that is different for each chemical species $t_{j}$. In fact, the descriptor contains two sets of expansion coefficients; the first set is evaluated without distinguishing between chemical species, i.e., for $w_{t_{j}}=1$, to represent local structure information. The second set of coefficients is evaluated with species-specific weights to capture differences in the local chemistry. By combining both local structure and chemistry, changes in the atomic positions and in the chemical species can be clearly distinguished. The dimension of the Chebyshev descriptor does not depend on the number of chemical species, and thus compositions with many chemical species do not result in any computational overhead [132]. Gastegger et al. later introduced species weights also to Behler-Parrinello symmetry functions in the weighted atom-centered symmetry functions method [134], though this approach does not include a separate structure descriptor. As seen in Fig. 6b-c, the accuracy that ANN potentials with the Chebyshev descriptor can achieve is not significantly affected by an increasing number of chemical species.

\section{Training ANN potentials}

The training of ANN potentials is the process of optimizing the weight parameters $\left\{a_{i}\right\}$ and $\{b\}$ in Eq. 1 for all artificial neurons. In the conventional ANN potential method, training on reference total energies from QM methods is most
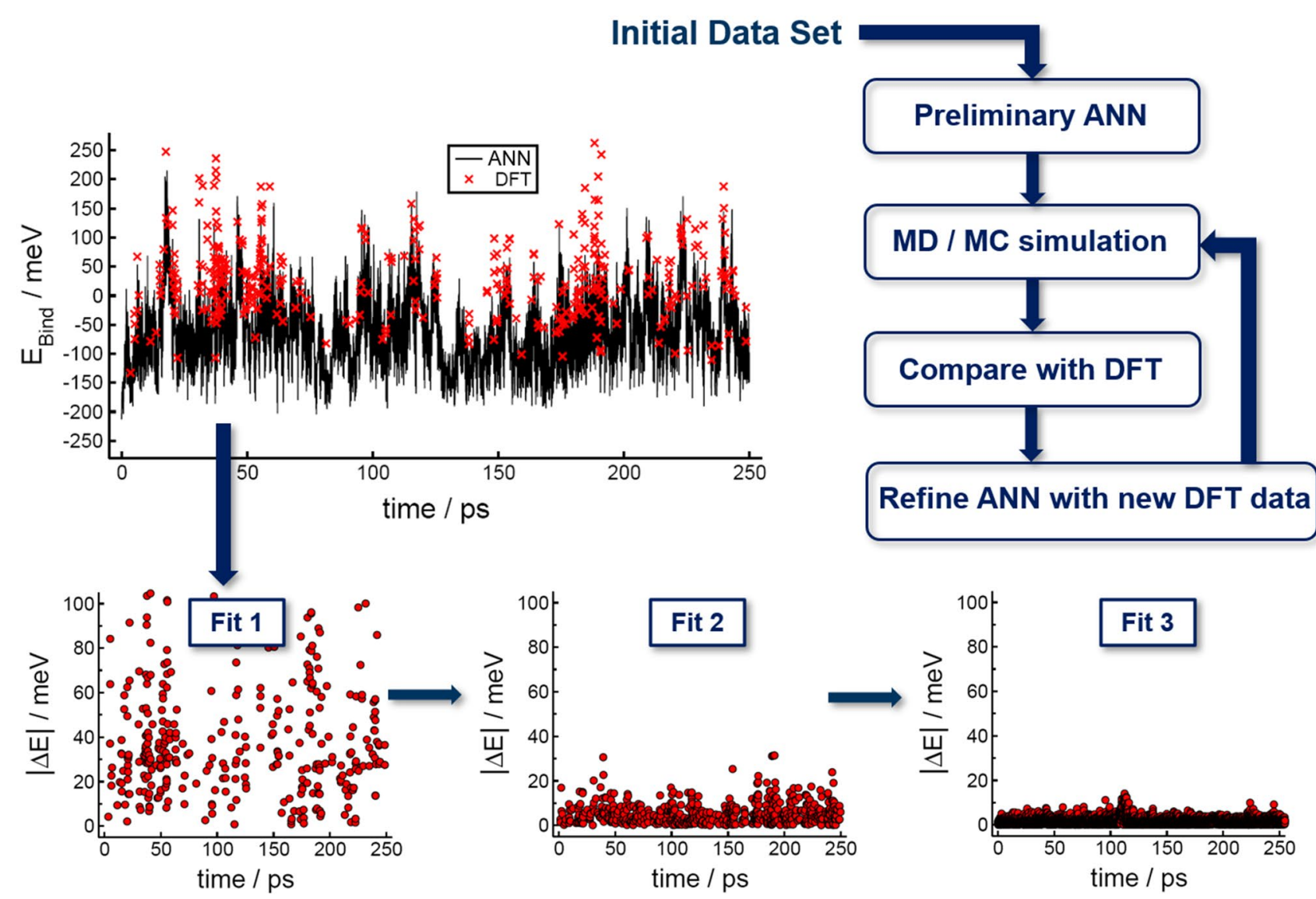

Fig. 7 Illustration of the systematic construction of ML potentials through the refinement of the reference data set in an active learning setup. The error $|\Delta E|$, i.e., the difference between the reference DFT and the ANN energies, for structures obtained in MD simulations decreases upon each iteration, from Fit 1 to Fit 3, as the sampling of the configurational space improves. (Adapted with permission from Ref. [102]) 
efficient, though approaches for the training of interatomic forces $[130,135]$ in addition to the energy have been developed. Chmiela et al. developed an alternative approach in which the forces are the only optimization target that uses an energy conservation criterion to avoid overfitting [136].

Irrespective of the training method, the reference data set is of critical importance for the transferability of ANN potentials. To guarantee complete reference data, active learning approaches are usually employed to systematically improve the data set [99]. A schematic of such an iterative refinement is shown in Fig. 7.

The principal idea behind active learning techniques is to make use of preliminary ANN potentials for the sampling of underrepresented structures. As such, oftentimes an initial data set is constructed based on chemical intuition, for example, by modification of ideal crystal structures or molecular geometries through scaling or deformation [127]. A preliminary ML potential is trained on this initial data and used in MC or MD simulations related to the eventual target application. A subset of the sampled structures is compared with the QM reference method, and if the discrepancy between the ANN prediction and the reference is too large, the structure is added to the reference data set. By repeating this procedure multiple times, the ANN potential becomes increasingly robust and transferable.

We outline here a basic active learning strategy but note that advanced techniques that improve the structure selection step are currently a very active field of research.

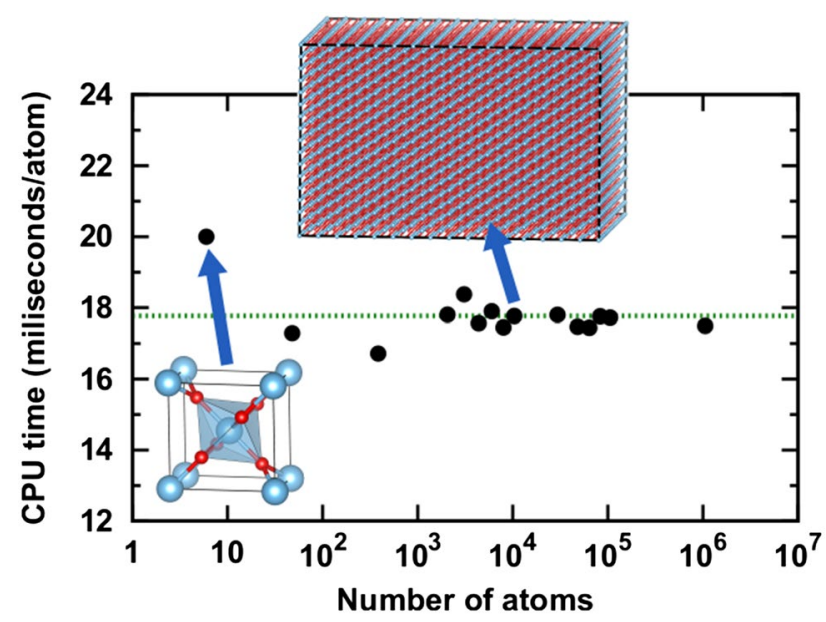

Fig. 8 The computational complexity of ANN potentials scales linearly with the number of atoms. The plot shows the evaluation time per atom as function of the number of atoms for periodic $\mathrm{TiO}_{2}$ structures with increasing size up to one million atoms. (Reproduced with permission from [127])

\section{Overview of MLP methods and implementations}

Thanks to the decomposition of the total energy into atomic contributions, Eq. (2), the computational complexity of the ANN potential method scales linearly with the number of atoms and can be implemented in efficient computer code (Fig. 8). Robust and easy-to-use public implementations have been emerging over recent years. Since the field of ML methods for atomistic simulations is advancing rapidly and new software implementations, descriptors, ML approaches, and training frameworks are published at a high rate, we refer here to a collection of publicly available tools and databases that will be continuously updated to include the most recent developments in the field: https://github.com/atomi sticnet/tools-and-data.

\section{Applications to industry}

The previous chapter has summarized the methodological advances that now enable to apply ML approaches to complex molecular and materials systems under realistic conditions. In the remainder of this review we will discuss recent examples in which MLPs and ML models are used to accelerate the calculation of industrially relevant properties with focus on drug discovery and materials design. A selection of the obtained properties, compared to their corresponding reference values where available, is summarized in Table 1 .

\section{Drug discovery applications}

In this section we discuss the use ML potentials and models for the prediction of properties that are relevant for drug discovery with the focus on two types of applications: the calculation of free energies and the prediction of spectroscopic properties.

A major challenge in calculating these properties lies in the complexity of the involved systems. The employed models have to be able to accurately describe small molecules, large molecular crystals and proteins interacting with small ligands in a solvent. These systems are governed by a diverse set of interactions between many different chemical elements involving diverse bonding types and (potentially) chemical reactivity. In contrast to simpler materials system, large units cells are required which makes it challenging to perform QM calculations. This is not only a problem for obtaining energies and forces required to perform MD or MC simulations but also for calculating other observables that are not (or only approximately) available from force fields. QM calculations in principle allow to calculate molecular dipoles, polarizabilities, and chemical shifts for simulating infrared (IR), Raman, and NMR spectra. Having access to these spectra allows to link experimentally observed spectral features to 
Table 1 Examples of properties calculated from machine learning (ML) potential simulations or using ML models based on quantum mechanical reference data compared to reference values, where available

\begin{tabular}{|c|c|c|c|c|c|}
\hline Property & System & ML Prediction & Reference value & Year & Refs. \\
\hline \multicolumn{6}{|l|}{ Drug discovery } \\
\hline Reaction free energy & Glycine proton transfer & $7.7 \mathrm{kcal} / \mathrm{mol}$ & DFT: $8.1 \mathrm{kcal} / \mathrm{mol}$ & 2018 & [137] \\
\hline Reaction barrier & Glycine proton transfer & $9.9 \mathrm{kcal} / \mathrm{mol}$ & DFT: $10.2 \mathrm{kcal} / \mathrm{mol}$ & 2018 & [137] \\
\hline \multirow[t]{7}{*}{ Solvation free energy } & Acetic acid & $-7.3 \mathrm{kcal} / \mathrm{mol}$ & DFT: $-7.5 \mathrm{kcal} / \mathrm{mol}$ & 2019 & [138] \\
\hline & Acetamide & $-11.7 \mathrm{kcal} / \mathrm{mol}$ & DFT: $-12.1 \mathrm{kcal} / \mathrm{mol}$ & 2019 & [138] \\
\hline & Acetone & $-3.9 \mathrm{kcal} / \mathrm{mol}$ & DFT: $-4.3 \mathrm{kcal} / \mathrm{mol}$ & 2019 & [138] \\
\hline & Benzene & $-0.6 \mathrm{kcal} / \mathrm{mol}$ & DFT: $-0.6 \mathrm{kcal} / \mathrm{mol}$ & 2019 & [138] \\
\hline & Ethanol & $-4.6 \mathrm{kcal} / \mathrm{mol}$ & DFT: $-4.8 \mathrm{kcal} / \mathrm{mol}$ & 2019 & [138] \\
\hline & Methylamine & $-2.5 \mathrm{kcal} / \mathrm{mol}$ & DFT: $-5.2 \mathrm{kcal} / \mathrm{mol}$ & 2019 & [138] \\
\hline & Aqueous LiF pair & $-231.5 \mathrm{kcal} / \mathrm{mol}$ & Exp.[139]: $-232.9 \mathrm{kcal} / \mathrm{mol}$ & 2020 & [140] \\
\hline \multicolumn{6}{|l|}{ Li-ion batteries } \\
\hline & Amorphous silicon anode & & & & \\
\hline Li diffusivity & $\mathrm{a}-\mathrm{Li}_{x} \mathrm{Si}(0.75<x<3.50)$ & $10^{-14}-10^{-10} \mathrm{~cm}^{2} \mathrm{~s}^{-1}$ & $\begin{array}{l}\text { Exp.[141-144]: } \\
10^{-14}-10^{-10} \mathrm{~cm}^{2} \mathrm{~s}^{-1}\end{array}$ & 2019 & [145] \\
\hline \multirow[t]{11}{*}{ Activation energy } & $\mathrm{a}-\mathrm{Li}_{x} \mathrm{Si}(0.75<x<3.50)$ & $0.5-0.8 \mathrm{eV}$ & N/A & 2019 & [145] \\
\hline & $\mathrm{a}-\mathrm{Li}_{x} \mathrm{Si}(0.02<x<0.06)$ & $1.21-1.46 \mathrm{eV}$ & Exp.[146]: $1.38-1.46 \mathrm{eV}$ & 2020 & [147] \\
\hline & Solid electrolytes & & & & \\
\hline & Amorphous- $\mathrm{Li}_{3} \mathrm{PO}_{4}$ & $0.55 \mathrm{eV}$ & Exp.[148]: $0.58 \mathrm{eV}$ & 2017 & [149] \\
\hline & $\mathrm{Li}_{10} \mathrm{GeP}_{2} \mathrm{~S}_{12}$ & $0.16 \mathrm{eV}$ & Exp.[150]: $0.22 \mathrm{eV}$ & 2020 & [151] \\
\hline & $\mathrm{Li}_{7} \mathrm{La}_{3} \mathrm{Zr}_{2} \mathrm{O}_{12}$ & $0.2-0.22 \mathrm{eV}$ & Exp.[152]: $0.21-0.22 \mathrm{eV}$ & 2020 & [151] \\
\hline & Cathode coating materials & & & & \\
\hline & $\mathrm{Li}_{2} \mathrm{~B}_{7} \mathrm{O}_{12}$ & $0.56 \pm 0.05 \mathrm{eV}$ & N/A & 2020 & [153] \\
\hline & $\mathrm{Li}_{3} \mathrm{Sc}_{2}\left(\mathrm{PO}_{4}\right)_{3}$ & $0.62 \pm 0.04 \mathrm{eV}$ & Exp.: [154] $0.65 \mathrm{eV}$ & 2020 & [153] \\
\hline & $\mathrm{Li}_{2} \mathrm{~B}_{6} \mathrm{O}_{9} \mathrm{~F}_{2}$ & $0.79 \pm 0.10 \mathrm{eV}$ & Exp.: [155] $0.92 \mathrm{eV}$ & 2020 & [153] \\
\hline & $\mathrm{LiCl}$ & $1.11 \pm 0.13 \mathrm{eV}$ & Exp.: [156] $0.83 \mathrm{eV}$ & 2020 & [153] \\
\hline
\end{tabular}

$x$ is the relative lithium content in the amorphous Li-Si alloys and varies during battery charge and discharge

their corresponding molecular motion which in turn enables to identify molecular structures.

As discussed in the previous section, the application of MLPs to complex systems with diverse chemical environments has now become possible with new methodological improvements including more general descriptors for multicomponent systems, automated training set generation, and force training. Even though these new approaches have just been developed (and continue to being extended) there are already several impactful applications of QM-based MLPs and ML models applied to bio-molecular systems relevant for the drug discovery process. Here we highlight some recent examples covering the investigation of chemical reactions in solution and solvation processes, the extension of force fields and semi-empirical QM/MM methods for simulations with improved accuracy, and the prediction of spectroscopic properties for the characterization of molecules and molecular crystals.

\section{Reaction and solvation free energies}

As discussed in the introduction, knowledge of binding free energies [22-24] allows to estimate relative binding affinities of a series of ligands and to rank them accordingly. Free energies are also central to understanding reaction mechanisms and transport processes. Solvation free energies determine the transport of a drug molecule to the target, which involves traveling through both aqueous media (blood) and lipophilic media (membranes) followed by desolvation before forming a ligand-protein complex [157]. The main challenges for obtaining reliable free energy values are insufficient sampling times and an inaccurate description of the PES.

Shen and Yang[137] employed ANNs to improve the accuracy of free energy calculations for two chemical reactions in solution, an $\mathrm{S}_{N} 2$ reaction and the intramolecular proton transfer reaction for glycine in water. Since chemical bonds are broken and formed during the process, a quantum mechanical description of the system for example within a QM/MM setup is required. To lower the computational 


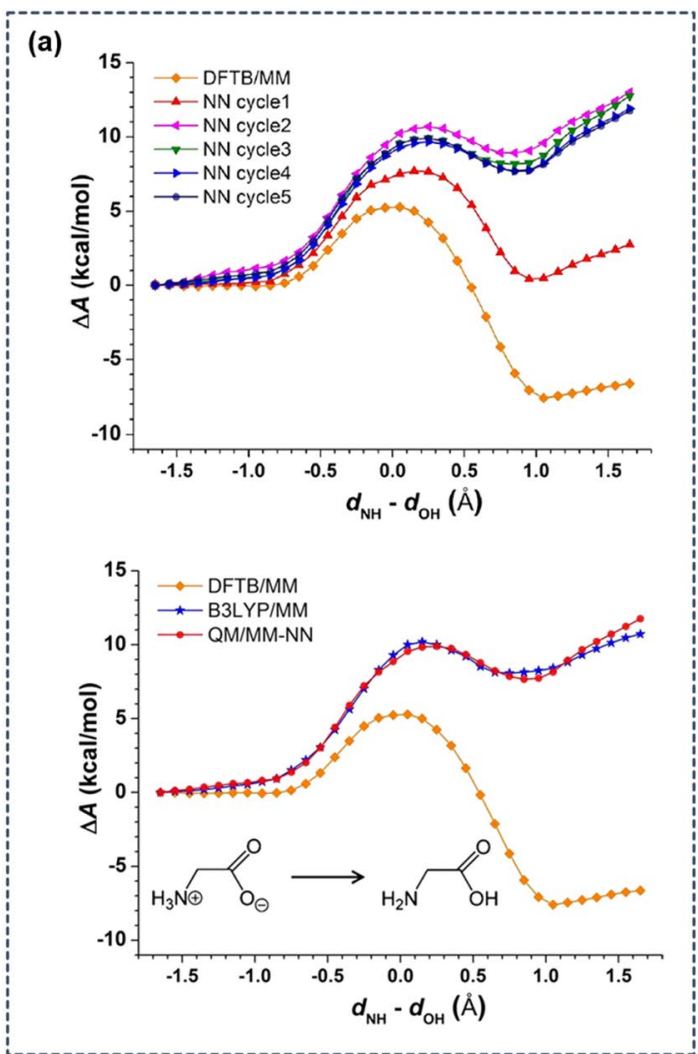

Fig. 9 Machine learning simulations for free energy calculations: a Intramolecular proton transfer reaction of glycine in water by Shen and Yang [137] using a QM/MM-NN setup in which an MLP is iteratively trained (top) to represent the energy difference between a low-level (DFTB) and a high-level (B3LYP) QM method. In the final iteration (bottom) the MLP correctly predicts the zwitterionic glycine tautomer as the predominant form, improving on the inaccurate description with the low-level method. b Solvation free energy of LiF

effort, the QM part can be replaced by a semi-empirical (SQM) method such as scc-DFTB [158] which results in a more efficient but less accurate description of the system. As shown in Fig. 9a the neutral form of aqueous glycine is incorrectly predicted as the dominant one. To improve on that, the authors developed the QM/MM-NN approach in which the energy difference between the lower-level SQM method and the high-level QM method is predicted by an ANN potential. An earlier example of such a composite strategy in which an ML correction is added to a computationally efficient but less accurate QM method is the deltamachine learning approach by Ramakrishnan et al. [159]. An important ingredient of the approach by Shen and Yang is the use of an adaptive procedure in which an initial MLP is iteratively improved by new structures, selected when the ANN input variables are outside of their training set boundaries. Using the QM/MM-NN setup with the MLP correction term, in each iteration of the potential the description of the free energy along the reaction coordinate improves until in water by Jinnouchi et al. [140] obtained from MLP-accelerated simulations trained on only the thermodynamic endpoints. The top panels show snapshots from thermodynamic integration simulations that correspond to the fully interacting system (left) and the system at small interactions (right), respectively. In the bottom panel paircorrelation functions of LiF in water obtained from the MLP (black line) are compared to results from QM simulations (red dashed line). A comparison of the ion solvation free energies is reported in Table 1

with the 5th iteration it is closely aligned with the high-level QM result (see Fig. 9a and Table 1) and correctly predicts the zwitterionic form as having the lowest free energy. For the glycine system, the difference MLP leads to a total CPU time for the QM/MM-NN MD simulations that is only about $1-8 \%$ of the time required to perform simulations with the conventional QM/MM setup, yielding an increase in efficiency by a factor of $10-100$. This comparison already includes the additional computational cost of running the QM reference calculations and training the MLP.

In a follow-up paper Zhang et al. [138] calculated solvation free energies for six small organic molecules (see Table 1) with an extended approach that addresses two challenges, (1) the identification of insufficiently sampled reference structures, and (2) the re-optimization process of the model after new structures were added to the training set. The selection criterion used in the previous work is based on the descriptor boundaries and by that has the potential to miss new data points that could lie inside the 
boundary region but be still very different from the current training structures. The authors therefore explored other approaches based on the energy range and different clustering algorithms. The training process was improved by using the component-wise gradient boosting algorithm [160] as a method to re-optimize the model with new data rather than each time training new models from scratch. These improvements enabled further time savings compared to the previous approach, requiring shorter simulations time and fewer additional structures for obtaining converged potentials. While in the extended approach a linear regression model was employed it will be interesting to see if an extension to more complex ML models such as ANNs can lead to further improvements.

Jinnouchi et al. [140] made use of a previously developed learn-on-the-fly ML approach $[161,162]$ to calculate solvation free energies of aqueous $\mathrm{LiF}$ ions at low computational cost. The solvation free energies of ions in water determine the properties of electrolyte solutions and greatly impact $\mathrm{pK}_{a}$ values [163] and protein stability [164]. Ion solvation free energies can in principle be obtained from QM simulations [165] by methods like thermodynamic perturbation theory (TPT) [166] or thermodynamic integration (TI) [166, 167] but have large error bars. In an TI approach one can perform a coupling constant integration from a reference system comprising of the non-interacting ion pair in solvent to a system where all atoms fully interact (see Fig. 9b). The authors now employed a variant of the Gaussian Approximation Potential (GAP) approach [87] with the Smooth Overlap of Atomic Positions (SOAP) descriptor [88] for the on-thefly generation of an MLP $[161,162]$ to speed up the TI simulations and obtain converged results. This approach employs self-learning to reduce the need for human intervention by using Bayesian inference to identify structures with high uncertainties which where then recalculated with the reference QM method and used to refine the MLP. A keys feature of the TI approach is that is requires only model training for the thermodynamic end points (the non-interacting and the fully interacting system). As shown in Table 1, the final free energy values obtained with the ML approach agree closely with the experimental values and also with results from QM simulations [163] while 10 times longer simulation times could be employed to reduce error bars and obtain converged values. Since the method is general and applicable to different systems it could be extended to obtain molecular solvation energies.

\section{Spectroscopic techniques for structure characterization}

Combining experimental measurements with QM calculations of infra red (IR), Raman, or NMR spectra is a powerful way to characterize the structure of molecular systems. Such combined approaches can for example help to identify the crystal structure of drugs that are available in form of molecular crystals which is the case for many marketed drugs. Understanding their crystalline form is crucial since it has an impact on several important properties such as stability, solubility, and bioavailability [173]. ML methods can help in two ways to improve the calculation of spectroscopic properties from atomistic simulations. They can (1) be used to represent the observables (dipoles, polarizabilities, NMR nuclear shifts) that are the ingredient for obtaining the spectrum and whose calculation by QM methods is often the computational bottleneck. Here, the structures for which the ML model predicts the spectrum are generated by performing a regular MM- or QM-based simulation. In approach (2), MLPs are trained to represent the PES of the system of interest and then efficiently generate the structures on which QM calculations are performed to calculate the spectroscopic property. Carrying out QM-calculations in such a post-processing step has the benefit that these calculations can be performed in parallel, as opposed to a purely QMbased approach in which a continuous trajectory has to be generated with a small time step and the observables are calculated on-the-fly. Approaches (1) and (2) can also be combined in a setup where the simulation is performed by an MLP and an additional ML model is used to represent the QM-trained spectroscopic observable. Here we discuss a number of recent examples in which ML approaches are applied to obtain spectroscopic properties for organic molecules in the gas phase, hydrogen-bonded liquids, and molecular solids.

IR spectra for bio-molecular systems are often obtained from static calculations with a normal mode analysis based on the harmonic approximation, thus neglecting important anharmonic and temperature effects. QM-based simulations allow to include these dynamic effects (and also reactive proton transfer events) but at high computational costs. A composite ML approach for the calculation of anharmonic IR spectra was developed by Gastegger et al. [168] in which the need for explicit QM calculations is fully circumvented by combining MLPs with an ML model to represent molecular dipoles. They employed ANNs to represent the PES, making use of an adaptive scheme for selecting new structures and training on atomic forces [174] which allowed the use of a small number of QM training points ( $\sim 700$ for the alanine tripeptide). To be able to train the MLP on large systems, a fragmentation approach was used in which large molecules are divided into smaller fragments for which reference QM calculations are more feasible. The molecular dipole moments were modelled by another ANN representing environment-dependent atomic charges. Since atomic charges are no observables, there is no unique way to calculate them. The solution used by the authors was to use the total dipole moment and molecular charge of the entire system as the target property. Equivalent to the atomic 

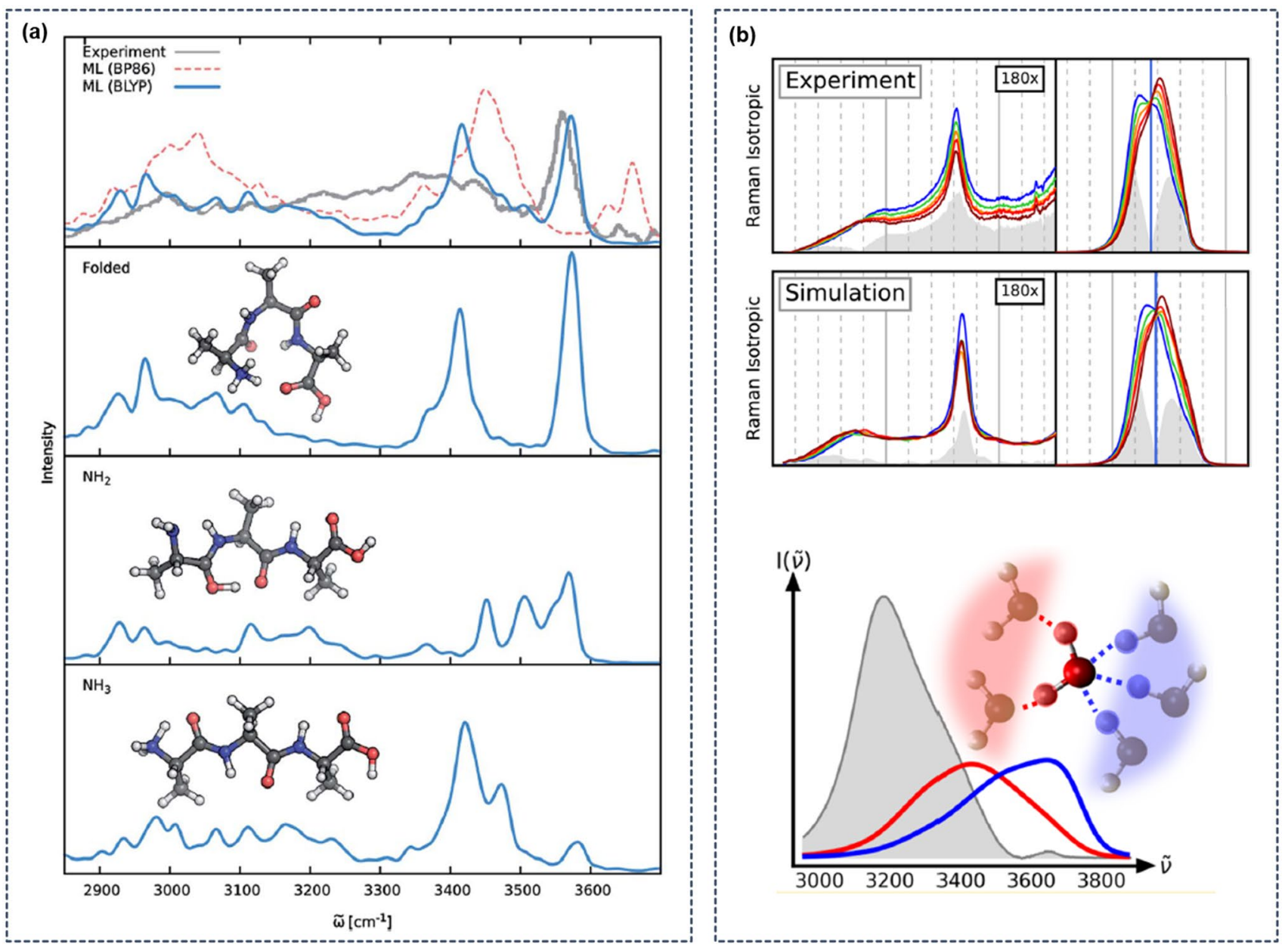

Fig. 10 Machine learning prediction of spectroscopic properties: a IR spectrum of the protonated alanine tripeptide by Gastegger et $a l$. [168] obtained from a composite ML approach in which the interatomic potential and the molecular dipoles are represented by individual ML models (Reproduced with permission from Ref. [168]Published by The Royal Society of Chemistry). In the top panel, the calculated spectrum obtained from ML models representing two different QM methods (BP86 and BLYP) is compared to the experimental spectrum [169]. The bottom panels show spectral contributions from the three main conformers. b Temperature-dependent

energies which are the output of atomic ANNs and in sum give the total energy of the system, the atomic charges can be seen as latent variables. The ML model that represents the environment-dependent charges therefore acts as a datadriven partitioning scheme without any constraints other then to match the target. Among other organic molecules, the protonated alanine tripeptide was used as test system to evaluate the ability of the combined ML approach to describe anharmonic, conformational, and dynamic effects, including proton transfer events, that all contribute to the IR spectrum (see Fig. 10a). While it was found that the resulting IR spectra show a strong dependence on the QM reference method, the efficiency of the ML approach (with timings of
Raman spectra of liquid water by Morawietz et al. [170, 171] calculated from MLP simulations and compared to experimental measurements. As shown in the top panels (Reprinted with permission from Ref. [170]. Copyright 2018 American Chemical Society), MLP-based simulations are able to accurately capture subtle spectral features like the bimodal $\mathrm{OH}$ stretching region and allow to identify molecules in overcoordinated environments by linking vibrational motion to structural parameters (bottom panel, Reprinted with permission from Ref. [171]. Copyright 2019 American Chemical Society)

1 hour instead of $>100$ days on a single CPU for obtaining the full spectrum) allows to quickly benchmark different QM methods to find the most suitable one for the system at hand. The authors also suggested that ANN-learned atomic charges could not only be used to obtain vibrational spectra but also for the augmentation of classical force fields, a route that was taken in the following application.

Kato et al. [175] constructed ML models to predict accurate charges for three proteins based on fragment molecular orbital (FMO) calculations. Commonly used force fields use fixed atomic charges and therefore neglect electronic polarization. Since the force field charges cannot adjust to a changing environment the description of the molecular 
recognition process between a protein and a ligand molecule might suffer. To address this issue, the authors trained an ANN model to learn atomic partial charges from QM calculations that take into account electronic polarization. They faced the challenging task to perform QM reference calculations for three complete proteins (polyQ10, Trip-Cage, and BRD2) containing up to 111 amino acids. Similar to the work by Gastegger et al. this was addressed by employing a fragmentation approach with the FMO method. Elementspecific ANNs using atom-centered symmetry functions where then trained to learn atomic charges from restrained electrostatic potential (RESP) calculations. In future applications, ML charge models could be combined with force fields to develop simulation methods that take into account polarization effects for the improved description of proteinligand interactions. Energies and atomic charges from FMO calculations of 1074 proteins were made freely available by the authors.

Returning to ML applications for the simulation of vibrational spectra, Morawietz et al. [170, 171] simulated temperature-dependent Raman spectra for liquid water using MLPs. They initially employed a variant of approach (2) in which trajectories at different temperatures where generated from ML-based MD simulations. QM calculations were then performed on these structures to obtain the polarizabilities required to calculate Raman spectra. As shown in Fig. 10b (top), these spectra accurately reproduce experimental measurements across the full liquid temperature range. In a second step, the authors bypassed the use of QM calculations and used the vibrational density of states (VDOS), obtained from the atomic velocities, as a proxy for the vibrational Raman features. In an combined effort with experimental decomposition techniques they made use of the VDOS to identify the structural origin of subtle vibrational features in the Raman spectrum. This analysis could for example identify the vibrational fingerprints of molecules residing in over-coordinated hydrogen-bond environments, species that play an important role in the transport of protons through membranes and the coordination of hydrophobic groups (see Fig. 10b, bottom).

In a complementary approach, Raimbault et al. [172] predicted anharmonic Raman spectra of paracetamol using QM simulations to generate the trajectories and an ML model to predict polarizabilities. They compared different GPR methods to learn polarizability and susceptibility of molecules and molecular crystals for reference data from QM calculations using density-functional perturbation theory (DFPT) calculations. DFPT results for anharmonic vibrational Raman spectra of molecular crystals were taken from prior work and made available in the NOMAD database $[176,177]$. DFPT calculations are typically four times more expensive then evaluating the forces during an MD simulation [177]. A symmetry-adapted GPR version
(SA-GPR) [178] was found to be most suitable for describing tensorial properties such as polarizabilities. The SAGPR approach has been also successfully applied to the prediction of Raman and IR spectra for liquid water and ice based on path integral MD simulations that include nuclear quantum effects [179]. Using an ensemble of $16 \mathrm{ML}$ models to estimate uncertainties, Raimbault et al. applied their approach to calculate the Raman spectrum of two crystal forms of paracetamol (see Fig. 11). Impressively, the ML model trained only on crystal form I is able to accurately predict the spectral lineshape for form II, even though the low-frequency modes that correspond to the intermolecular interactions vary considerably between the two forms. The high degree of transferability demonstrates the benefit of using a local approach in which total polarizabilities are decomposed into atom-centered contributions based on local environments. While this approach still relies on QM simulations, we expect to see an increase in the number of "MLonly" approaches (as in Ref. [168]]) where MLPs are used to perform MD simulations and ML models (for example based on GPR) represent observables like dipole moments and polarizabilities to obtain accurate spectra with greatly reduced computational costs.

In the final example by Paruzzo et al. [180], NMR chemical shifts for molecular crystals were predicted by ML models based on the GPR approach. Chemical shifts are key data for determining structure and dynamics of bio-molecular systems and can for example help to identify the protonation state of enzyme active sites [181]. While many empirical tools have been developed to aid in the the assignment of experimental NMR spectra they are often optimized for a small subset of systems and neglect dynamical effects. Calculating chemical shift with QM methods $[182,183]$ has a more general validity for different chemical environments [184]. Combining QM calculations with NMR measurements enable chemical shift-based crystallography for validating the structure of molecular solids [185, 186]. Paruzzo et al. employed a GPR framework with the SOAP kernel [88] to learn DFT chemical shifts for structures from the CSD database [187] with estimated uncertainties based on a previously introduced resampling scheme [188]. The ML model was trained on 500 structures randomly sampled from a CSD subset containing 61,000 structures that are small enough ( $<200$ atoms) to perform QM calculations and then applied to calculate chemical shifts of six molecular crystals comprising of up to 1500 atoms. The authors then demonstrated that, without making use of experimental chemical shifts, their method is accurate enough to correctly determine the structure of two molecular solids: cocaine and the drug AZD8329. This application again exemplifies the benefit of employing local ML models: they can be transferable to larger systems without loss of accuracy and in addition scale linear with system size. Calculating the full set of 


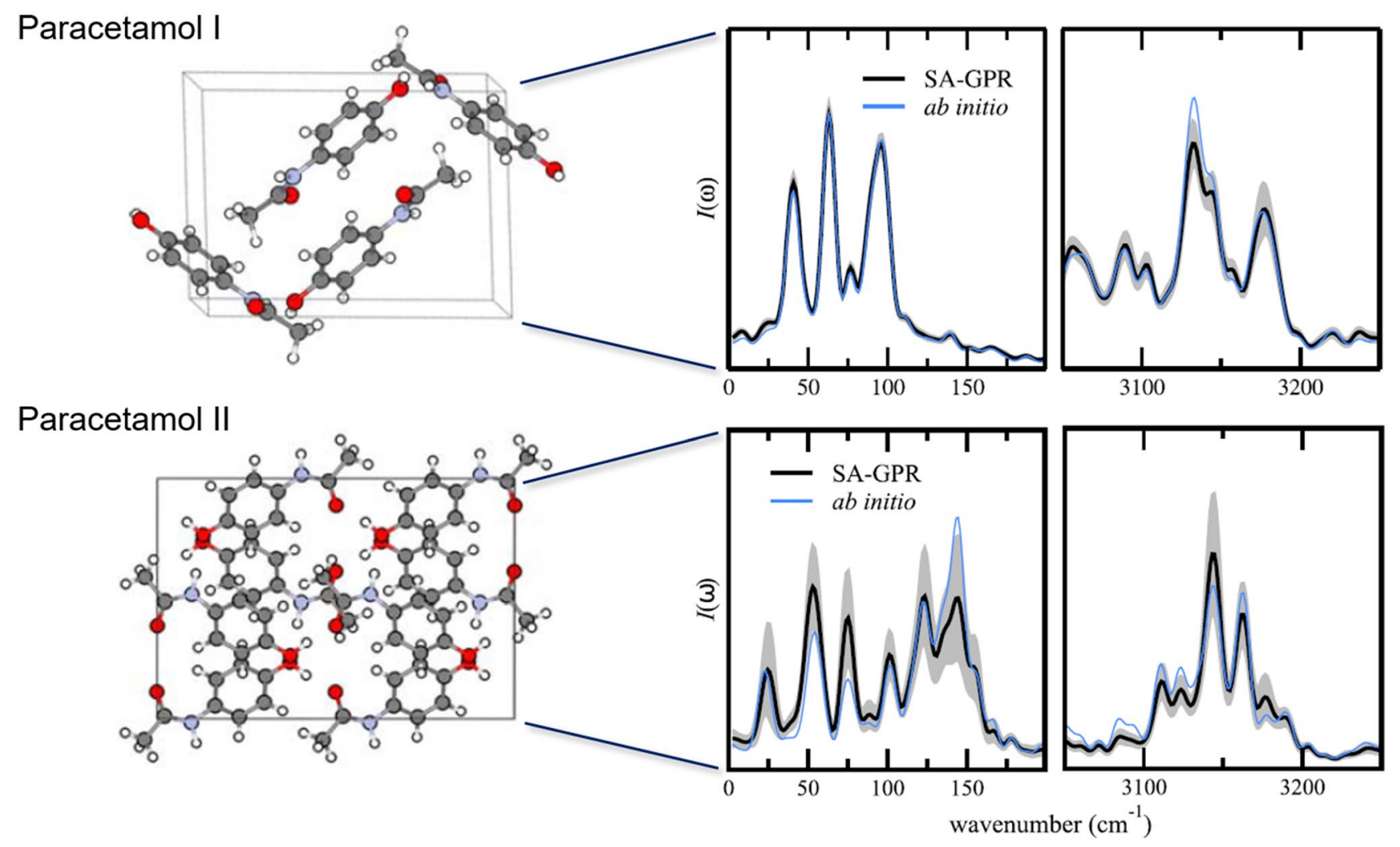

Fig. 11 Machine learning prediction of spectroscopic properties: Anharmonic Raman spectra of the Paracetamol crystal in forms I and II by Raimbault et al. [172] calculated with an ML model (SA-GPR) of the polarizability tensor trained on form I only. The top panels show the low- and high-frequency parts of the Raman spectrum for form I compared to the reference QM results (ab initio). ML results

chemical shifts for six molecular crystals took a few minutes with the ML model which, for the largest model, is a speedup of a factor of $10^{6}$ compared to a direct QM approach. The ML tool to predict NMR chemical shifts for the elements ${ }^{1} \mathrm{H}$, ${ }^{13} \mathrm{C},{ }^{15} \mathrm{~N},{ }^{17} \mathrm{O}$ and ${ }^{33} \mathrm{~S}$ is publicly available [189].

\section{Materials discovery applications}

In correspondence to drug discovery applications, the application of ML models to materials discovery has also seen a steep rise of research activity during the last decade, owing to the availability of methods, public implementations, and increased computer power [190]. In this section, we review some of the recent successful applications in the area with a focus on inorganic solid materials.

\section{Phase diagram predictions}

Before considering any other properties of a potential functional material, the first requirement for computational were obtained from an ensemble of 16 models from which uncertainties have been estimated (shaded area). The results for form II in the bottom panels demonstrate the high transferability of the ML model which can accurately represent the overall lineshape of the unseen molecular crystal

materials design is the ability to predict whether a hypothetical compound is stable. Predicting the products of organic synthesis (such as drugs) requires knowledge of reaction kinetics. In contrast, the stability of inorganic solids is mostly governed by thermodynamics [191]. As such, in good approximation, predicting the likely stability of a novel material is equivalent with predicting the thermodynamic phase diagram. This approximation can be further improved by considering also the kinetics of nucleation, e.g., by modeling the nucleation and growth of inorganic phases.

Brute-force atomistic calculations of phase diagrams are challenging because the time scales on which phase transitions occur are often not achievable with QM simulation methods. Here, ML potentials can be used as a dropin replacement in some cases. For example, Morawietz et al. simulated the melting of ice with MD simulations using ANN potentials [114], revealing the importance of $\mathrm{vdW}$ interactions for a correct description of the mechanism of the phase transition. Robinson et al. also used ML potential based MD simulations to determine the phase diagram of potassium as a function of the pressure and 


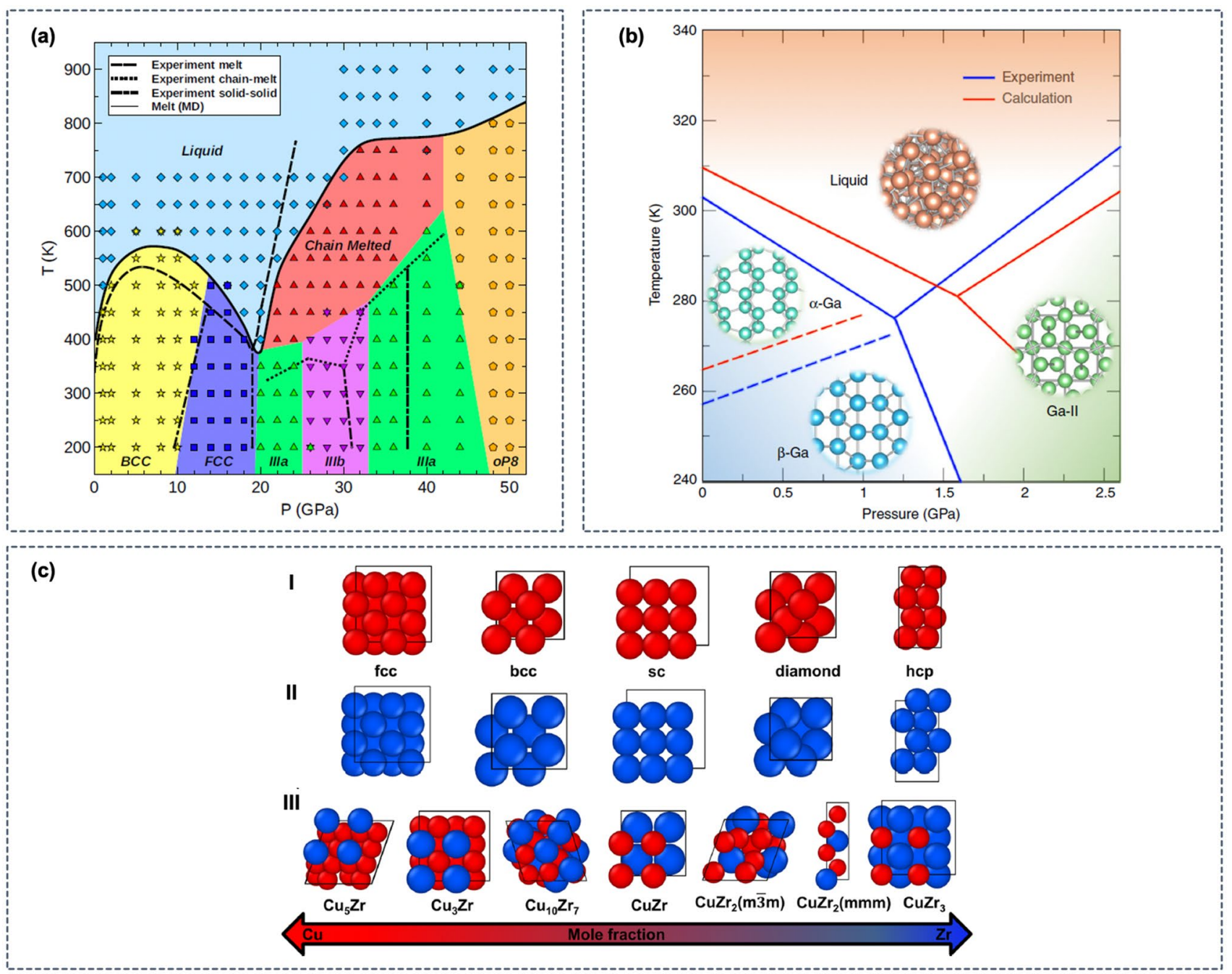

Fig. 12 ML-based simulations for the exploration of phase diagrams of inorganic materials. a Temperature and pressure dependent phase diagram of potassium obtained from MD simulations using an ML potential [192]. Each point in the figure represents the result from an individual ML-based MD simulation in the NVT statistical ensemble. Symbols distinguish between different equilibrium phases. (Reproduced with permission from [192]). b Phase diagram of gallium nucleation from the melt using metadynamics MD simulations with

temperature [192], which exhibits a complex chain-melted phase that had previously not been characterized in detail. The MD simulations by Robinson et al. were initialized in the expected ground-state phase for a given pressure at $200 \mathrm{~K}$, and MD simulations in the $N V T$ statistical ensemble were used to simulate phase transitions with temperature. The resulting phase diagram is shown in Fig. 12a.

While the process of melting is challenging to model, the reverse, i.e., crystallization from the melt, typically occurs at even longer timescales owing to nucleation barriers. In few cases, rapid crystallization can be modeled with direct MD simulations. Sosso et al. investigated the fast crystallization of $\mathrm{GeTe}$, a phase-change compound, from the supercooled an ML potential [193]. The predicted phase diagram (red lines) is compared to the experimentally measured phase diagram (blue lines). c Crystal structures of the CuZr alloys and of the $\mathrm{Cu}$ and $\mathrm{Zr}$ constituents used for training of an ANN potential by Andolina et al. [194]. The ANN potential trained on the crystalline phases was shown to predict the properties of the amorphous $\mathrm{CuZr}$ alloy with remarkable accuracy

liquid using ML potential MD simulations and identified the atomic-scale mechanism responsible for the rapid nucleation rate [195]. The same system was also investigated by Gabardi et al. who observed the nucleation of crystalline GeTe in 3 ns long melt-quench MD simulations with an ANN potential [196], finding that a crystallization mechanism similar to that in supercooled liquids can be achieved.

For many materials, phase transitions between solid phases or crystallization cannot be modeled with direct MD simulations as the time scale remains inaccessible despite the speed-up from ML potentials. In such cases, ML potentials in combination with accelerated MD techniques, such as the metadynamics approach by Parrinello 
and coworkers [54, 197], have been successfully employed. Behler and Parrinello modeled the polymorphic phase transitions in elemental Si using metadynamics [198], and a similar approach was used by Eshet et al. for the construction of the $P-T$ phase diagram of elemental sodium [199]. Bonati and Parrinello investigated crystallization of silicon from the melt with well-tempered metadynamics [197] using an ANN potential [200], identifying a single collective variable derived from the Debye structure factor to steer the crystallization. A related approach was employed by Niu et al. for the calculation of the phase diagram for gallium nucleation from the melt [193]. Gallium exhibits a complex phase behavior owing to the mixed covalent and metallic bonding, making the element a challenging benchmark case for phase diagram calculations. As seen in Fig. 12b, the phase diagram predicted by accelerated ML potential MD simulations is in excellent agreement with the experimental reference, demonstrating that ML potentials are sufficiently flexible to capture the complex atomic interactions of elemental gallium.

ML potentials have also been used for the modeling of polymorphism and phases with variable compositions in compounds that consist of multiple chemical species. In two separate studies, Artrith and coworkers showed that an ANN potential can accurately reproduce the stability of different $\mathrm{ZnO}$ [96] and $\mathrm{TiO}_{2}$ [127] polymorphs. Kong et al. used ANN potentials and an ML-augmented sampling technique to determine the phase diagram of $\mathrm{CoO}$ phases with varying Co:O ratio [201]. ANN potentials have been also used to model multicomponent alloys with varying composition, such as the $\mathrm{AuCu}$ alloys, both in the bulk and in nanoparticles [202, 203].

Apart from crystalline phases, the increased efficiency of ANN potentials compared to DFT calculations makes modeling disordered or amorphous phases accessible, which generally require larger structure models than crystal structures. Artrith et al. employed a combination of an evolutionary algorithm and an ANN potential to determine the phase diagram of the amorphous LiSi alloys [204]. In this study, the amorphous phase was explicitly sampled and characteristic structural motifs were included in the reference data set for the ANN potential training. Recently, Andolina et al. [194] trained an ANN potential on the crystalline $\mathrm{CuZr}$ alloy phases (Fig. 12c) and demonstrated that the resulting potential can accurately predict the properties of amorphous $\mathrm{CuZr}$ phases as well, which is a remarkable display of the transferability that ML potentials can achieve.

\section{Properties of catalyst materials}

The design and discovery of novel materials for heterogeneous catalysis is an area of great relevance for the chemical industry [206], and QM calculations for the computational prediction of the properties of catalyst materials are well established [207]. However, most computational studies make use of simplified catalyst models, such as single-crystal surfaces in vacuum, whereas catalytically active sites may in reality depend on the environment and on defects in the catalyst material. The greater computational efficiency of ML potentials has enabled the modeling of more realistic catalytic conditions and materials in recent years. Here, we focus again on applications of ANN potentials. Other ML applications for catalysis have recently been reviewed by Goldsmith et al. [208] and by Kitchin [209].

ML potential simulations have been of particular use for the modeling of non-idealized catalyst structures. For example, Artrith et al. constructed an ANN potential for the simulation of $\mathrm{ZnO}$-supported $\mathrm{Cu}$ nanoparticles [100], the catalyst for methanol synthesis [210, 211], and investigated the dynamic structure changes of the catalyst at 1,000 K using MD simulations. Such large-scale MD simulations would not have been possible with first principles QM methods, and conventional interatomic potentials would not have been able to capture the mixed metallic and ionic bonding in the $\mathrm{Cu} / \mathrm{ZnO}$ interface region.

Even unsupported catalyst nanoparticles are often beyond the length-scale limit of QM methods, especially when extensive sampling of atomic configurations is needed. Artrith and Kolpak showed that ANN potentials trained on surface structures and cluster configurations of $\mathrm{CuAu}$ alloys can reproduce the surface phase diagrams and Wulff shapes of the different alloys as a function of the chemical potentials (Fig. 13a) [202]. These ANN potentials were then used in large-scale $\mathrm{MC}$ simulations to determine low-energy atomic orderings in nanoparticles with up to 6500 atoms and in $\mathrm{AuCu}$ clusters and surfaces in contact with water, which showed the strong impact of water on the alloy surface termination and could explain the catalytic activity of $\mathrm{CuAu}$ nanoalloys for $\mathrm{CO}_{2}$ reduction [202]. The same authors also employed ANN potentials to investigate the temperature-dependent dynamics of $\mathrm{CuAu}$ nanoparticles in grandcanonical $(\mu V T)$ MD simulations [203]. The phase diagrams and the stability region of icosahedral $\mathrm{CuAu}$ nanoparticles compared to nanoparticles with truncated face-centered cubic shape are shown in Fig. 13b. Kolsbjerg et al. demonstrated for small $\mathrm{Pt}_{13}$ clusters how a combination of an ANN potential and an evolutionary algorithm can be used for the search for low-energy cluster structures [212], finding that a thermal ensemble of low-energy structures provides a better description for the catalyst than the zero temperature ground state structure alone. Sun and Sautet also used an evolutionary optimization strategy coupled with an ANN potential to determine the structures of Pt nanoparticles in hydrogen-rich atmosphere [205], which revealed a complex interplay of the Pt particles with the hydrogen gas resulting in a rich distribution of thermally accessible metastable $\mathrm{Pt}$ nanoparticles with very different properties. Example 

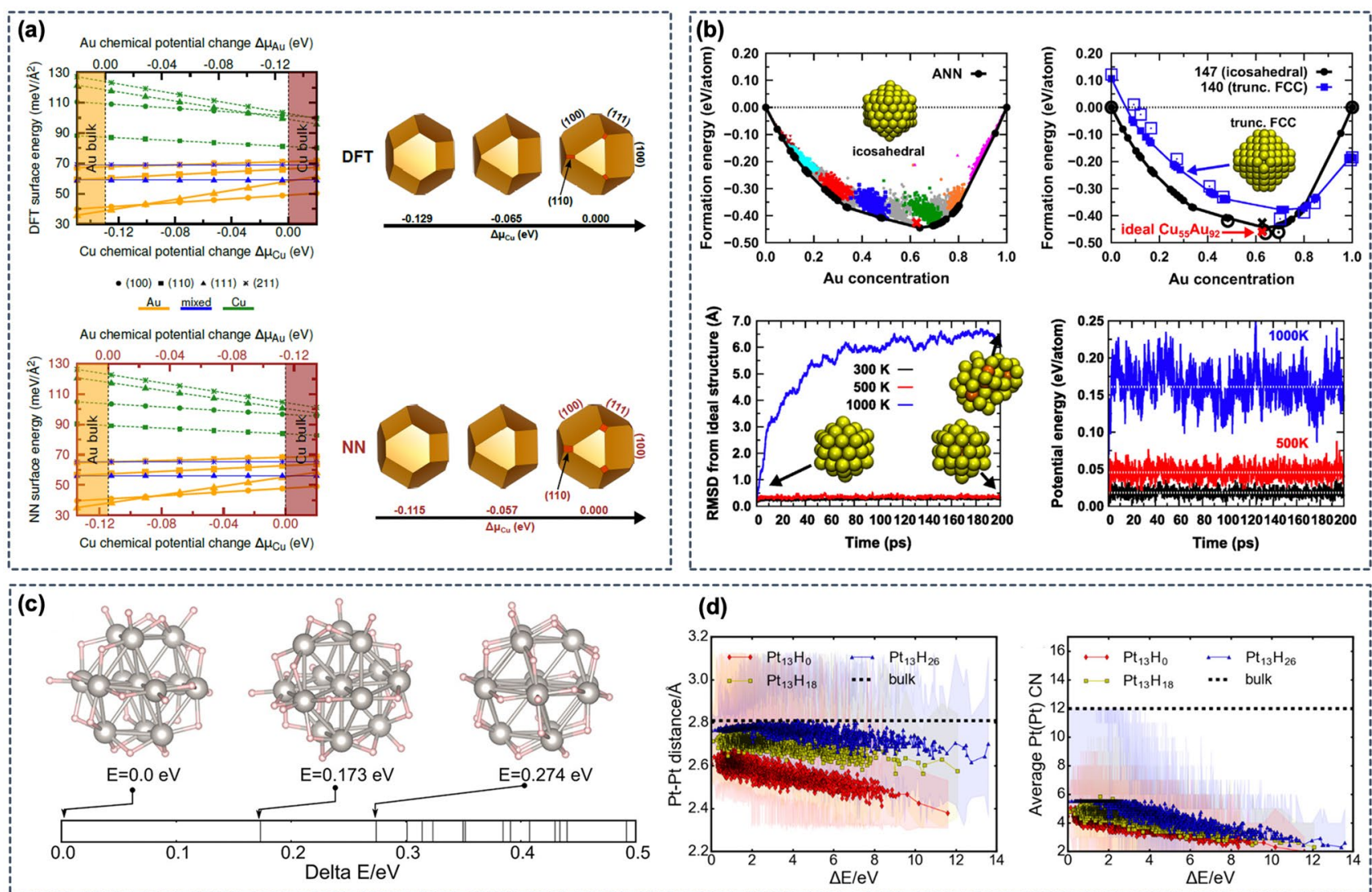

(d)
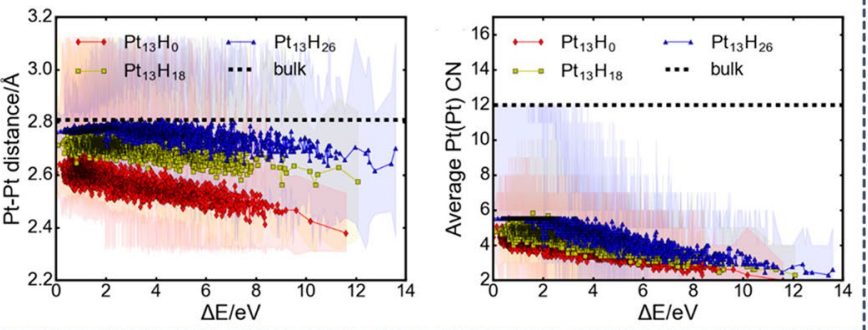

Fig. 13 ML potential simulation of catalyst materials: a surface phase diagrams of the of low-index surfaces of the $\mathrm{Cu}_{2} \mathrm{Au}_{2}$ alloy with different terminations ( $\mathrm{Au}, \mathrm{Cu}$, and mixed) as function of the $\mathrm{Au} /$ $\mathrm{Cu}$ chemical potentials, as predicted by DFT (top) and by an ANN potential (bottom). Symbols denote different facets, and surface terminations are indicated by line types and colors (yellow $=$ Au terminated, blue $=$ mixed, green $=\mathrm{Cu}$ ). Exemplary Wulff constructions corresponding to three different chemical potentials are also shown. (Reproduced with permission from Ref. [202]) b Formation energies

particle configurations and statistics of the Pt-Pt nearestneighbor distribution and $\mathrm{Pt}$ coordination numbers $(\mathrm{CN})$ are shown in Fig. 13c-d.

\section{Properties of battery materials}

The second major class of functional materials that has been investigated using ML potentials are materials for lithium ion batteries. Lithium ion batteries (LIB) consist essentially of two electrodes, the cathode and anode, that are submerged in or separated by electrolyte and are in contact with an external circuit. When an LIB is discharged (i.e., the battery is used), $\mathrm{Li}^{+}$cations are shuttled from the anode through the electrolyte to the cathode, and simultaneously electrons are released from the anode, perform work while they travel along the external circuit, and eventually arrive and convex hull construction for $\mathrm{CuAu}$ nanoparticles with 55 atoms. Different colors and point sizes indicate different chemical potentials used in grand canonical $(\mu V T)$ MC simulations. (Reproduced with permission from Ref. [203].). c Low-energy structures of Pt nanoparticles in hydrogen atmosphere. The energies of the particle structures are shown relative to the most stable configuration. Statistics of the Pt-Pt nearest neighbor distances and the average Pt coordination number as function of the relative energy are shown in panel (d). (Reproduced with permission from Ref. [205])

at the cathode as well. This process is reversed when the LIB is charged.

First principles QM calculations are widely used for the calculation of many properties of LIBs, such as the voltage and the electrochemical or thermal stability of the components [8]. However, QM based modeling is most practical for crystalline materials, although both non-crystalline electrode and electrolyte materials are of great relevance for LIBs. This limitation is especially significant for the investigation of Li transport in electrodes, the electrolyte, and interface regions, which can become rate limiting in LIBs. Recently, ANN potentials have enabled the simulation of Li diffusion in non-crystalline phases that had previously not been accessible.

For example, nanostructured amorphous silicon (a-Si) is a promising anode material for the next-generation of LIBs but its reaction with lithium has not been well understood 

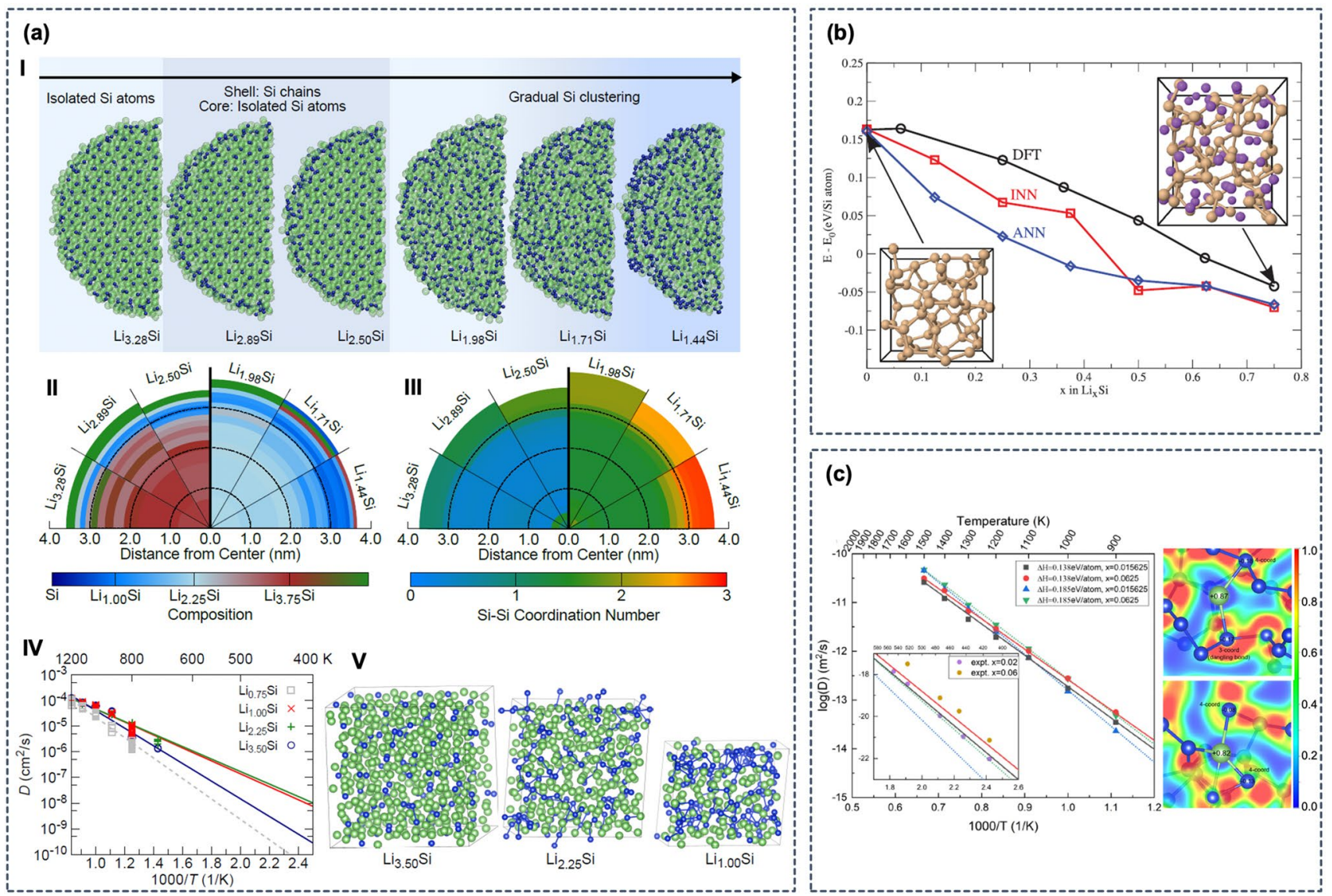

(c)

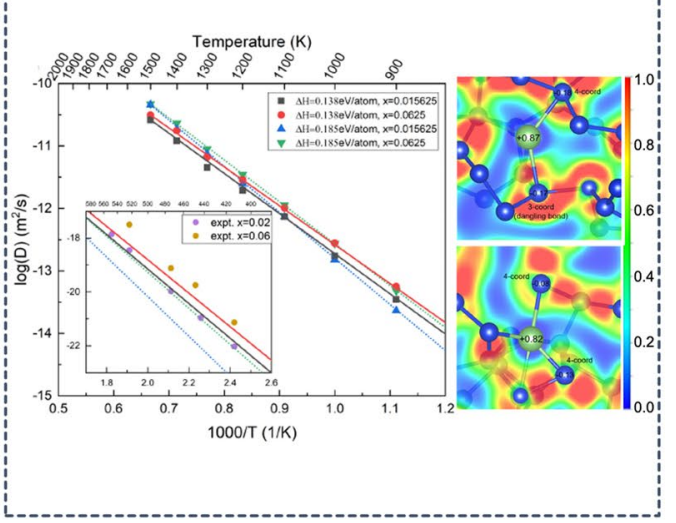

Fig. 14 Lithium transport in amorphous silicon anodes for lithiumion batteries. a Atomic structures of $\mathrm{Li}_{x} \mathrm{Si}$ alloy nanoparticle during delithiation (battery discharge) [145]. The change of the composition in the core of the $\mathrm{Li}_{x} \mathrm{Si}$ nanoparticles is shown in subfigure (a.I), and the change in the $\mathrm{Si}$ coordination numbers are shown in (a.II and a.III). Panels (a.IV) and (a.V) show an Arrhenius plot with the temperature-dependent lithium diffusivity in bulk amorphous LiSi alloys and representative bulk structures for different $\mathrm{Li}: \mathrm{Si}$ ratios, respectively. b Formation energies of amorphous LiSi structures as predicted by two different ANN potential approaches (ANN and INN, implanted neural networks) compared to the DFT reference energies [213]. (c) Arrhenius plot for Li diffusion in different amorphous silicon structures (left) and visualization of the electron localization function (ELF) for different structural motifs in the amorphous LiSi, $\mathrm{Li}$ bonding to an undercoordinated $\mathrm{Si}$ atom (top) and $\mathrm{Li}$ bonding to a fully coordinated $\mathrm{Si}$ atom (bottom). The numbers indicate the Bader charges of the $\mathrm{Li}$ and $\mathrm{Si}$ atoms. (Reproduced with permission from reference [147]) on the atomic scale [215]. Artrith et al. employed a combination of ANN potentials and an evolutionary algorithm to model electrochemical amorphization and to sample the phase diagram of the amorphous LiSi alloys [204]. The resulting ANN potential (based on a QM database reported in Ref. [216]) was subsequently used by the same authors to investigate $\mathrm{Li}$ transport in realistic $\mathrm{LiSi}$ nanoparticles containing up to 12,000 atoms including the amorphization and the change of the Li diffusivity upon delithiation (Fig. 14aI-III) [145]. One conclusion from these simulations is that the distribution of $\mathrm{Si}$ atoms within the structure strongly affects the Li diffusion, and Li rich regions are beneficial for Li diffusion (Fig. 14aIV-V). Onat et al. also trained ANN potentials for the modeling of amorphous LiSi structures [213]. The authors proposed an implanted neural network (INN) approach, in which the ANN potential is first pre-trained on the individual components $(\mathrm{Li}, \mathrm{Si})$ before it is used for the amorphous LiSi alloys (Fig. 14b). Li transport in the amorphous LiSi alloys was also investigated by Li et al. using ANN potential based MD simulations [147], also finding a strong dependence of the Li diffusivity on the local Si environment. Using the structure models from ANN potential simulations, the authors performed an electronicstructure analysis of the bonding in the atomic structures with DFT, finding that undercoordinated Si atoms interact more strongly with $\mathrm{Li}$ atoms and can impede Li diffusion (Fig. 14c), which is in agreement with the observations by Artrith et al. [145].

$\mathrm{Li}$ transport is not only important in electrode materials, but also in the electrolyte and in electrode-electrolyte interphases. Solid-state batteries (SSB) are a class of prospective high-energy-density LIBs in which the conventional liquid 

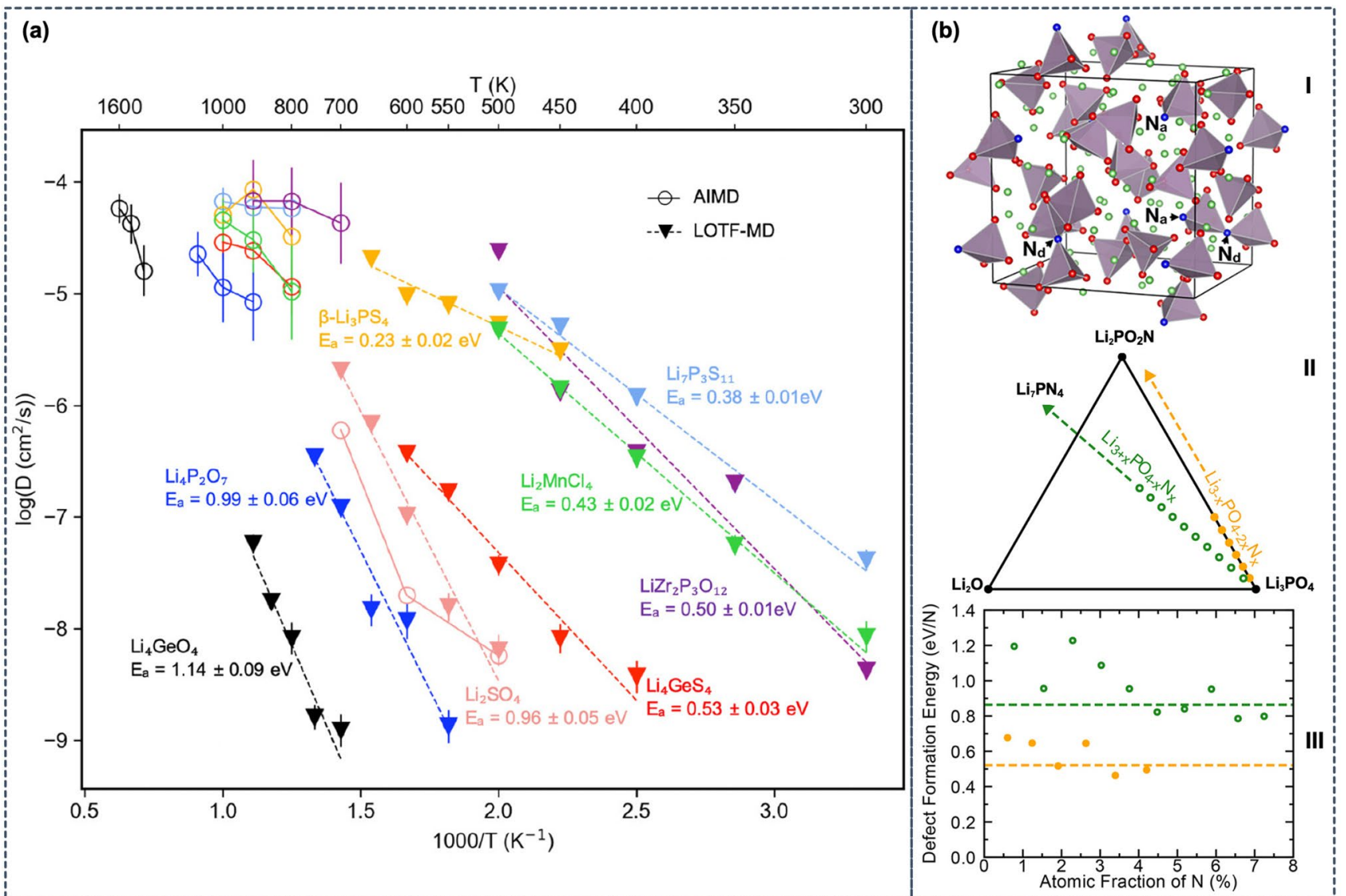

Fig. 15 Machine learning simulations for solid-state batteries: a Arrhenius plot with $\mathrm{Li}$ diffusivities obtained from ab initio MD (AIMD) simulations using a learning-on-the-fly (LOTF) ML potential based on the MTP method [153]. The ML potential simulations make low temperatures accessible that are closer to room temperature, whereas conventional AIMD simulations are limited to very high temperatures that are not relevant for battery operation. (Reproduced with permission from Ref. [153]) b Representative structure (I) and DFT phase diagram (II) of LiPON near-ground-state crystal structures [214]. Two different composition lines for nitrogen dop-

electrolytes are replaced with solid $\mathrm{Li}$ ion conductors [217, 218]. The Li transport in such solid electrolytes and across electrode/electrolyte interfaces is crucial for the performance of SSBs.

In recent work, ML potentials have been employed to investigate $\mathrm{Li}$ diffusion in crystalline and non-crystalline solid electrolytes, which are otherwise challenging to model with QM methods. Wang et al. used an MTP based ML potential to carry out long MD simulations of Li diffusion in eight different prospective coating materials for electrodes in SSB [153]. The authors made use of an on-the-fly learning approach to accelerate QM based ab initio MD simulations and to enable simulating long time scales of up to 2 nanoseconds. Fig. 15 shows an Arrhenius plot with a comparison of the Li diffusivities from AIMD, which have large uncertainties due to the limited sampling, with the more accurately ing are indicated in yellow (Li replacement) and green ( $\mathrm{Li}$ addition), respectively. Panel (III) shows the corresponding defect formation energies for nitrogen doping, as calculated with ANN-potential augmented sampling and DFT calculations. All defect structures are predicted to be unstable with respect to decomposition into $\mathrm{Li}_{2} \mathrm{O}$, $\mathrm{Li}_{3} \mathrm{PO}_{4}$, and $\mathrm{Li}_{2} \mathrm{PO}_{2} \mathrm{~N}$, showing that amorphous LiPON is metastable. Nitrogen doping via $\mathrm{Li}$ replacement is thermodynamically favored over doping with $\mathrm{Li}$ addition. (Reproduced with permission from Ref. [214])

determined diffusivities from ML potential simulations. The study also identified cases for which the Arrhenius law did not hold up to the temperatures accessible by DFT, demonstrating that long MD simulations at low temperatures are needed to observe the relevant diffusion behavior.

Amorphous lithium phosphate $\left(\mathrm{Li}_{3} \mathrm{PO}_{4}\right)$ is a $\mathrm{Li}$ ion conductor with potential applications as solid electrolyte in allsolid batteries [219], that has attracted much interest because of its chemical and electrochemical stability. Li, Watanabe et al. employed ANN potential based MD simulations to investigate $\mathrm{Li}$ transport in amorphous $\mathrm{Li}_{3} \mathrm{PO}_{4}$ [149], considering also large structure models with up to $\sim 1,000$ atoms and $\mathrm{Li}$ off-stoichiometries. The activation energies for $\mathrm{Li}$ diffusion were estimated to be $\sim 0.55 \mathrm{eV}$, in good agreement with experiment. Nitrogen-doped amorphous $\mathrm{Li}_{3} \mathrm{PO}_{4}$ (LiPON) exhibits better Li conductivity than pristine $\mathrm{Li}_{3} \mathrm{PO}_{4}$ 
and was investigated using a combination of ANN potentials and DFT calculations by Lacivita et al. [214]. The authors employed an ANN-potential augmented sampling approach with an evolutionary algorithm to determine low-energy amorphous LiPON structure models, which were subsequently recomputed with DFT to ensure accuracy. Fig. 15b shows a representative structure model and the DFT formation energies for different amounts of $\mathrm{N}$ doping. The study concluded that amorphous LiPON is generally metastable and decomposition into $\mathrm{Li}_{2} \mathrm{O}, \mathrm{Li}_{3} \mathrm{PO}_{4}$, and $\mathrm{Li}_{2} \mathrm{PO}_{2} \mathrm{~N}$ is thermodynamically favored. The comparison of two different reaction pathways for $\mathrm{N}$ doping showed, furthermore, that $\mathrm{N}$ substitution with simultaneous Li removal is energetically most likely.

Another example of transport simulations using ANN potentials is the work by $\mathrm{Li}$ et al. who modeled $\mathrm{Cu}$ diffusion in amorphous $\mathrm{Ta}_{2} \mathrm{O}_{5}$ [220]. In this study, the ANN potential was trained only on the energy differences caused by $\mathrm{Cu}$ intercalation, thereby reducing the complexity of the potential energy surface [220].

While the direct modeling of ionic diffusion with ML potentials is a powerful approach to investigate transport mechanisms, the computational screening for novel ionic conductors does not necessarily require the full complexity of atomistic diffusion simulations. We note, therefore, that ML has also been proposed for the discovery of solid-state $\mathrm{Li}$ ion conductors without explicit simulation. Two examples of such materials discovery applications are a study based on unsupervised learning by Zhang et al. [221] and a transferlearning approach applied to billions of candidate materials by Cubuk et al. [222].

\section{Remaining challenges and outlook}

There are still several remaining challenges in the construction and applications of QM-based ML approaches that we expect to be addressed in future developments. Specifically, the construction of ML models (1) still relies on manual validation to ensure reliability and transferability, and (2) requires large data sets from QM calculations that may incur computational overheads.

The construction of ML models such as ML potentials requires large reference data sets that come with a computational overhead. It is therefore important to decide first whether a specific research question can be directly addressed with QM based calculations. An ML model is only cost-effective if that is not possible, or if the cost of the QM calculations would exceed the cost of producing the reference data and training an ML model. Note that some applications require length or time scales that cannot directly be accessed with QM methods but may be investigated using more efficient ML models because of the linear scaling of their computational cost (Fig. 8), such as the modeling of nanoparticles reviewed in Sects. "Spectroscopic techniques for structurecharacterization" and "Properties of catalyst materials".

It is important to keep in mind that ML models are only as good as the reference data that they were trained on, and, for example, ML potentials trained on DFT data will generally exhibit the same inaccuracies as the original DFT method.

Careful validation is needed, since the flexibility of the employed ML approaches leads to poor performance in describing data that lies outside the trained range, which can result in stability issues when new regions or conditions are explored. Possible solutions are the inclusion of additional local information (such as forces, curvatures, electronegativity, etc.) and physical constraints in the training process or the use of automated frameworks that generate only relevant structures that improve the description of configurations at the boundary of the training region.

Another challenge is data scarcity due to the high computational cost of the QM reference calculations, especially in the case of unstructured systems that cannot be easily described by simplified models, such as proteins in solution. A partial solution already used in several of the discussed applications is to employ fragmentation approaches in which large molecules are divided into smaller fragments for which QM calculations are more feasible [168, 175, 223]. Transfer learning techniques can also be used to reduce the number of reference calculations, for example by training a model to a more efficient lower-level method first before re-training on a smaller data set obtained from a more expensive higher-level method [224]. Other possibilities are the use of multitask techniques, in which generalization performance is improved by simultaneously training on multiple related tasks, which could be for example applied to the spectroscopy models summarized in Sect. "Spectroscopic techniques for structurecharacterization".

Finally, one could also imagine to completely circumvent the need to run converged MD simulations and employ hybrid approaches in which an ML model learns to predict a converged property from a small number of MD snapshots [225].

Despite the remaining challenges, the impressive applications reviewed in the previous chapter demonstrate that QMbased ML approaches can now be applied to the complex systems required to simulate realistic processes of industrial relevance. It is now possible to obtain a diverse set of properties such as solvation free energies, vibrational spectra, phase diagrams, and transport coefficients with increased efficiency and accuracy, approaching the top left corner in Fig. 1. The rapidly growing number of ML simulations and models, most of which have just been published in the last few years, is a consequence of significant methodological 
advances, including transferable descriptors and automated training procedures, and the availability of open-source tools. Additionally, community efforts have given rise to public repositories that facilitate the exchange of ML models and data sets. We compiled an extensible list of public tools, data sources, and repositories at https://github.com/atomi sticnet/tools-and-data. Together, these resources offer exciting opportunities for knowledge transfer and for exploration of new ML applications in academia and in industry.

Acknowledgements We thank Alexander Urban for commenting on the manuscript.

Open Access This article is licensed under a Creative Commons Attribution 4.0 International License, which permits use, sharing, adaptation, distribution and reproduction in any medium or format, as long as you give appropriate credit to the original author(s) and the source, provide a link to the Creative Commons licence, and indicate if changes were made. The images or other third party material in this article are included in the article's Creative Commons licence, unless indicated otherwise in a credit line to the material. If material is not included in the article's Creative Commons licence and your intended use is not permitted by statutory regulation or exceeds the permitted use, you will need to obtain permission directly from the copyright holder. To view a copy of this licence, visit http://creativecommons.org/licenses/by/4.0/.

\section{References}

1. Jorgensen WL (2004) The many roles of computation in drug discovery. Science 303(5665):1813. https://doi.org/10.1126/scien ce.1096361

2. Van Drie JH (2007) Computer-aided drug design: the next 20 years. J Comput Aided Mol Des 21(10):591. https://doi. org/10.1007/s10822-007-9142-y

3. Aminpour M, Montemagno C, Tuszynski JA (2019) An overview of molecular modeling for drug siscovery with specific illustrative examples of applications. Molecules 24(9):1693. https://doi. org/10.3390/molecules24091693

4. Curtarolo S, Hart GLW, Nardelli MB, Mingo N, Sanvito S, Levy O (2013) The high-throughput highway to computational materials design. Nat Mater 12(3):191. https://doi.org/10.1038/nmat3 568

5. Oganov AR, Pickard CJ, Zhu Q, Needs RJ (2019) Structure prediction drives materials discovery. Nature Rev Mater 4(5):331. https://doi.org/10.1038/s41578-019-0101-8

6. Jain A, Shin Y, Persson KA (2016) Computational predictions of energy materials using density functional theory. Nature Rev Mater 1(1):1. https://doi.org/10.1038/natrevmats.2015.4

7. Seh ZW, Kibsgaard J, Dickens CF, Chorkendorff I, Nørskov JK, Jaramillo TF (2017) Combining theory and experiment in electrocatalysis: insights into materials design. Science. https://doi. org/10.1126/science.aad4998

8. Urban A, Seo DH, Ceder G (2016) Computational understanding of Li-ion batteries. NPJ Computat Mater 2(1), 1. https://doi. org/10.1038/npjcompumats.2016.2

9. Frenkel D, Smit B (2001) Understanding molecular simulation: from algorithms to applications. Elsevier, New York

10. Allen MP, Tildesley DJ (2017) Computer Simulation of Liquids. Oxford University Press, Oxford
11. Jorgensen WL, Tirado-Rives J (2005) Potential energy functions for atomic-level simulations of water and organic and biomolecular systems. Proc Nat Acad Sci 102(19):6665. https://doi. org/10.1073/pnas.0408037102

12. Becker CA, Tavazza F, Trautt ZT, Buarque de Macedo RA (2013) Considerations for choosing and using force fields and interatomic potentials in materials science and engineering. Curr Opin Solid State Mater Sci 17(6):277. https://doi.org/10.1016/j.cossm s.2013.10.001

13. Cornell WD, Cieplak P, Bayly CI, Gould IR, Merz KM, Ferguson DM, Spellmeyer DC, Fox T, Caldwell JW, Kollman PA (1995) A Second Generation Force Field for the Simulation of Proteins, Nucleic Acids, and Organic Molecules. J Am Chem Soc 117(19):5179. https://doi.org/10.1021/ja00124a002

14. MacKerell AD, Banavali N, Foloppe N (2000) Development and current status of the CHARMM force field for nucleic acids. Biopolymers 56(4):257 https://doi.org/10.1002/10970282(2000)56:4<257::AID-BIP10029>3.0.CO;2-W

15. Oostenbrink C, Villa A, Mark AE, Gunsteren WFV (2004) A biomolecular force field based on the free enthalpy of hydration and solvation: the GROMOS force-field parameter sets 53A5 and 53A6. J Comput Chem 25(13):1656. https://doi.org/10.1002/ jcc. 20090

16. Jorgensen WL, Tirado-Rives J (1988) The OPLS [optimized potentials for liquid simulations] potential functions for proteins, energy minimizations for crystals of cyclic peptides and crambin. J Am Chem Soc 110(6):1657. https://doi.org/10.1021/ja002 $14 \mathrm{a} 001$

17. Jones JE (1924) On the determination of molecular fields-II. From the equation of state of a gas. Proc R Soc Lond Ser A 106(738):463. https://doi.org/10.1098/rspa.1924.0082

18. Daw MS, Baskes MI (1983) Semiempirical, quantum mechanical calculation of hydrogen embrittlement in metals. Phys Rev Lett 50(17):1285. https://doi.org/10.1103/PhysRevLett.50.1285

19. Brenner DW (1990) Empirical potential for hydrocarbons for use in simulating the chemical vapor deposition of diamond films. Phys Rev B 42(15):9458. https://doi.org/10.1103/PhysR evB.42.9458

20. Mortier J, Rakers C, Bermudez M, Murgueitio MS, Riniker S, Wolber G (2015) The impact of molecular dynamics on drug design: applications for the characterization of ligand- macromolecule complexes. Drug Discov Today 20(6):686. https://doi. org/10.1016/j.drudis.2015.01.003

21. Ganesan A, Coote ML, Barakat K (2017) Molecular dynamics-driven drug discovery: leaping forward with confidence. Drug Discov Today 22(2):249. https://doi.org/10.1016/j.drudi s.2016.11.001

22. Christ CD, Mark AE, van Gunsteren WF (2010) Basic ingredients of free energy calculations: a review. J Comput Chem 31(8):1569. https://doi.org/10.1002/jcc. 21450

23. Chodera JD, Mobley DL, Shirts MR, Dixon RW, Branson K, Pande VS (2011) Alchemical free energy methods for drug discovery: progress and challenges. Curr Opin Struct Biol 21(2):150. https://doi.org/10.1016/j.sbi.2011.01.011

24. Homeyer N, Stoll F, Hillisch A, Gohlke H (2014) Binding free energy calculations for lead optimization: assessment of their accuracy in an industrial drug design context. J Chem Theory Comput 10(8):3331. https://doi.org/10.1021/ct5000296

25. Christ CD, Fox T (2014) Accuracy assessment and automation of free energy calculations for drug design. J Chem Inf Model 54(1):108. https://doi.org/10.1021/ci4004199

26. Kuhn B, Tichý M, Wang L, Robinson S, Martin RE, Kuglstatter A, Benz J, Giroud M, Schirmeister T, Abel R, Diederich F, Hert J (2017) Prospective evaluation of free energy calculations for the prioritization of Cathepsin L inhibitors. J Med Chem 60(6):2485. https://doi.org/10.1021/acs.jmedchem.6b01881 
27. Wagner V, Jantz L, Briem H, Sommer K, Rarey M, Christ CD (2017) Computational macrocyclization: from de novo Macrocycle generation to binding affinity estimation. ChemMedChem 12(22):1866. https://doi.org/10.1002/cmdc.201700478

28. Pérez-Benito L, Keränen H, van Vlijmen H, Tresadern G (2018) Predicting binding free energies of PDE2 inhibitors. The difficulties of protein conformation. Sci Rep 8(1):4883. https://doi. org/10.1038/s41598-018-23039-5

29. Yang Q, Burchett W, Steeno GS, Liu S, Yang M, Mobley DL, Hou X (2020) Optimal designs for pairwise calculation: an application to free energy perturbation in minimizing prediction variability. J Comput Chem 41(3):247. https://doi.org/10.1002/ jec. 26095

30. Shi Q, Izvekov S, Voth GA (2006) Mixed atomistic and coarsegrained molecular dynamics: simulation of a membranebound ion channel. J Phys Chem B 110(31):15045. https://doi. org/10.1021/jp062700h

31. Lonsdale R, Ward RA (2018) Structure-based design of targeted covalent inhibitors. Chem Soc Rev 47(11):3816. https://doi. org/10.1039/C7CS00220C

32. Martínez-Suárez L, Frenzel J, Marx D, Meyer B (2013) Tuning the reactivity of a $\$ \$ \mathrm{Cu} / \mathrm{ZnO} \$ \$$ nanocatalyst via gas phase pressure. Phys Rev Lett 110(8):086108. https://doi.org/10.1103/ PhysRevLett.110.086108

33. DCIS Inc. Daylight Theory: SMARTS - A Language for Describing Molecular Patterns. http://www.daylight.com/dayhtml/doc/ theory/theory.smarts.html (2020)

34. Mobley DL, Bannan CC, Rizzi A, Bayly CI, Chodera JD, Lim VT, Lim NM, Beauchamp KA, Slochower DR, Shirts MR, Gilson MK, Eastman PK (2018) Escaping atom types in force fields using direct chemical perception. J Chem Theory Comput 14(11):6076. https://doi.org/10.1021/acs.jctc.8b00640

35. Senftle TP, Hong S, Islam MM, Kylasa SB, Zheng Y, Shin YK, Junkermeier C, Engel-Herbert R, Janik MJ, Aktulga HM, Verstraelen T, Grama A, van Duin ACT (2016) The ReaxFF reactive force-field: Development, applications and future directions, npj Computational Materials 2(1):1. https://doi.org/10.1038/npjco mpumats.2015.11

36. Wang LP, Chen J, Van Voorhis T (2013) Systematic parametrization of polarizable force fields from quantum chemistry data. J Chem Theory Comput 9(1):452. https://doi.org/10.1021/ ct300826t

37. Huang L, Roux B (2013) Automated force field parameterization for nonpolarizable and polarizable atomic models based on ab initio target data. J Chem Theory Comput 9(8):3543. https://doi.org/10.1021/ct4003477

38. Car R, Parrinello M (1985) Unified approach for molecular dynamics and density-functional theory. Phys Rev Lett 55(22):2471. https://doi.org/10.1103/PhysRevLett.55.2471

39. Marx D, Hutter J (2009) Ab initio molecular dynamics: basic theory and advanced methods. Cambridge University Press, Cambridge

40. Burke K (2012) Perspective on density functional theory. J Chem Phys 136(15):150901. https://doi.org/10.1063/1.47045 46

41. Carloni P, Rothlisberger U, Parrinello M (2002) The role and perspective of $\mathrm{Ab}$ initio molecular dynamics in the study of biological systems. Acc Chem Res 35(6):455. https://doi.org/10.1021/ ar010018u

42. Marx D, Parrinello M (1996) Ab initio path integral molecular dynamics: basic ideas. J Chem Phys 104(11):4077. https://doi. org/10.1063/1.471221

43. Markland TE, Ceriotti M (2018) Nuclear quantum effects enter the mainstream. Nature Rev Chem 2(3):1. https://doi. org/10.1038/s41570-017-0109
44. Hobza P, Kabeláč M, Šponer J, Mejzlík P, Vondrášek J (1997) Performance of empirical potentials (AMBER, CFF95, CVFF, CHARMM, OPLS, POLTEV), semiempirical quantum chemical methods (AM1, MNDO/M, PM3), and ab initio Hartree-Fock method for interaction of DNA bases: Comparison with nonempirical beyond Hartree-Fock results, Journal of Computational Chemistry 18(9), 1136. https://doi.org/10.1002/(SICI)1096987X(19970715)18:9<1136::AID-JCC3>3.0.CO;2-S

45. Korth M, Thiel W (2011) Benchmarking semiempirical methods for thermochemistry, kinetics, and noncovalent interactions: OMx methods are almost as accurate and robust as DFTGGA methods for organic molecules. J Chem Theory Comput 7(9):2929. https://doi.org/10.1021/ct200434a

46. ̌ezáč J, Hobza P (2012) Advanced corrections of hydrogen bonding and dispersion for semiempirical quantum mechanical methods. J Chem Theory Comput 8(1):141. https://doi. org/10.1021/ct200751e

47. Kř́íz K, Řezáč J (2020) Benchmarking of semiempirical quantum-mechanical methods on systems relevant to computeraided drug design. J Chem Inf Model 60(3):1453. https://doi. org/10.1021/acs.jcim.9b01171

48. Elstner M (2006) The SCC-DFTB method and its application to biological systems. Theor Chem Acc 116(1):316. https://doi. org/10.1007/s00214-005-0066-0

49. Brandenburg JG, Grimme S (2014) Accurate modeling of organic molecular crystals by dispersion-corrected density functional tight binding (DFTB). J Phys Chem Lett 5(11):1785. https://doi. org/10.1021/jz500755u

50. Bannwarth C, Ehlert S, Grimme S (2019) GFN2-xTB - an accurate and broadly parametrized self-consistent tight-binding quantum chemical method with multipole electrostatics and densitydependent dispersion contributions. J Chem Theory Comput 15(3):1652. https://doi.org/10.1021/acs.jctc.8b01176

51. Schmitz S, Seibert J, Ostermeir K, Hansen A, Göller AH, Grimme S (2020) Quantum chemical calculation of molecular and periodic peptide and protein structures. J Phys Chem B 124(18):3636. https://doi.org/10.1021/acs.jpcb.0c00549

52. Aqvist J, Warshel A (1993) Simulation of enzyme reactions using valence bond force fields and other hybrid quantum/classical approaches. Chem Rev 93(7):2523. https://doi.org/10.1021/ cr00023a010

53. Svensson M, Humbel S, Froese RDJ, Matsubara T, Sieber S, Morokuma K (1996) ONIOM: A Multilayered Integrated MO + MM Method for Geometry Optimizations and Single Point Energy Predictions. A Test for Diels-Alder Reactions and $\mathrm{Pt}(\mathrm{P}(\mathrm{t}-$ Bu)3) $2+\mathrm{H} 2$ Oxidative Addition, The Journal of Physical Chemistry 100(50), 19357. https://doi.org/10.1021/jp962071j

54. Laio A, VandeVondele J, Rothlisberger U (2002) A Hamiltonian electrostatic coupling scheme for hybrid Car-Parrinello molecular dynamics simulations. J Chem Phys 116(16):6941. https:// doi.org/10.1063/1.1462041

55. Senn HM, Thiel W (2007) QM/MM studies of enzymes. Curr Opin Chem Biol 11(2):182. https://doi.org/10.1016/j. cbpa.2007.01.684

56. Kühne TD, Krack M, Mohamed FR, Parrinello M (2007) Efficient and accurate Car-Parrinello-like approach to Born-Oppenheimer molecular dynamics. Phys Rev Lett 98(6):066401. https ://doi.org/10.1103/PhysRevLett.98.066401

57. Wang LP, Song C (2019) Car-Parrinello monitor for more robust Born-Oppenheimer molecular dynamics. J Chem Theory Comput 15(8):4454. https://doi.org/10.1021/acs.jctc.9b00439

58. Rupp M, Tkatchenko A, Müller KR, von Lilienfeld OA (2012) Fast and accurate modeling of molecular atomization energies with machine learning. Phys Rev Lett 108(5):058301. https://doi. org/10.1103/PhysRevLett.108.058301 
59. von Lilienfeld OA, Müller KR, Tkatchenko A (2020) Exploring chemical compound space with quantum-based machine learning, Nature Reviews Chemistry pp. 1-12. https://doi.org/10.1038/ s41570-020-0189-9

60. Cuny J, Xie Y, Pickard CJ, Hassanali AA (2016) Ab initio quality NMR parameters in solid-state materials using a high-dimensional neural-network representation. J Chem Theory Comput 12(2):765. https://doi.org/10.1021/acs.jctc.5b01006

61. Lee J, Seko A, Shitara K, Nakayama K, Tanaka I (2016) Prediction model of band gap for inorganic compounds by combination of density functional theory calculations and machine learning techniques. Phys Rev B 93(11):115104. https://doi.org/10.1103/ PhysRevB.93.115104

62. Pilania G, Mannodi-Kanakkithodi A, Uberuaga BP, Ramprasad R, Gubernatis JE, Lookman T (2016) Machine learning bandgaps of double perovskites. Sci Rep 6(1):19375. https://doi. org/10.1038/srep19375

63. Zhuo Y, Mansouri Tehrani A, Brgoch J (2018) Predicting the band gaps of inorganic solids by machine learning. J Phys Chem Lett 9(7):1668. https://doi.org/10.1021/acs.jpclett.8b00124

64. Tran K, Ulissi ZW (2018) Active learning across intermetallics to guide discovery of electrocatalysts for $\mathrm{CO} 2$ reduction and $\mathrm{H}$ 2 evolution. Nature Catal 1(9):696. https://doi.org/10.1038/s4192 9-018-0142-1

65. Geiger P, Dellago C (2013) Neural networks for local structure detection in polymorphic systems. J Chem Phys 139(16):164105. https://doi.org/10.1063/1.4825111

66. Cendagorta JR, Tolpin J, Schneider E, Topper RQ, Tuckerman ME (2020) Comparison of the performance of machine learning models in representing high-dimensional free energy surfaces and generating observables. J Phys Chem B 124(18):3647. https ://doi.org/10.1021/acs.jpcb.0c01218

67. Wirnsberger P, Ballard AJ, Papamakarios G, Abercrombie S, Racanière S, Pritzel A, Rezende DJ, Blundell C (2020) Targeted free energy estimation via learned mappings, arXiv:2002.04913 [physics, stat]

68. Sanchez-Lengeling B, Aspuru-Guzik A (2018) Inverse molecular design using machine learning: generative models for matter engineering. Science 361(6400):360. https://doi.org/10.1126/ science.aat 2663

69. Schreck JS, Coley CW, Bishop KJM (2019) Learning retrosynthetic planning through simulated experience. ACS Central Sci 5(6):970. https://doi.org/10.1021/acscentsci.9b00055

70. Liu B, Ramsundar B, Kawthekar P, Shi J, Gomes J, Luu Nguyen Q, Ho S, Sloane J, Wender P, Pande V (2017) Retrosynthetic reaction prediction using neural sequence-to-sequence models. ACS Central Sci 3(10):1103. https://doi.org/10.1021/acscentsci $.7 \mathrm{~b} 00303$

71. Mayr A, Klambauer G, Unterthiner T, Steijaert M, Wegner JK, Ceulemans H, Clevert DA, Hochreiter S (2018) Large-scale comparison of machine learning methods for drug target prediction on ChEMBL. Chem Sci 9(24):5441. https://doi.org/10.1039/ C8SC00148K

72. Green DVS, Pickett S, Luscombe C, Senger S, Marcus D, Meslamani J, Brett D, Powell A, Masson J (2020) BRADSHAW: a system for automated molecular design. J Comput Aided Mol Des 34(7):747. https://doi.org/10.1007/s10822-019-00234-8

73. Artrith N, Lin Z, Chen JG (2020) Predicting the activity and selectivity of bimetallic metal catalysts for ethanol reforming using machine learning. ACS Catal 10(16):9438. https://doi. org/10.1021/acscatal.0c02089

74. Gómez-Bombarelli R, Wei JN, Duvenaud D, Hernández-Lobato JM, Sánchez-Lengeling B, Sheberla D, Aguilera-Iparraguirre J, Hirzel TD, Adams RP, Aspuru-Guzik A (2018) Automatic chemical design using a data-driven continuous representation of molecules. ACS Central Sci 4(2):268. https://doi.org/10.1021/ acscentsci.7b00572

75. Winter R, Montanari F, Noé F, Clevert DA (2019) Learning continuous and data-driven molecular descriptors by translating equivalent chemical representations. Chem Sci 10(6):1692. https ://doi.org/10.1039/C8SC04175J

76. Winter R, Montanari F, Steffen A, Briem H, Noé F, Clevert DA (2019) Efficient multi-objective molecular optimization in a continuous latent space. Chem Sci 10(34):8016. https://doi. org/10.1039/C9SC01928F

77. Butler KT, Davies DW, Cartwright H, Isayev O, Walsh A (2018) Machine learning for molecular and materials science. Nature 559(7715):547. https://doi.org/10.1038/s41586-018-0337-2

78. Elton DC, Boukouvalas Z, Fuge MD, Chung PW (2019) Deep learning for molecular design - a review of the state of the art. Mol Syst Des Engi 4(4):828. https://doi.org/10.1039/C9ME0 0039A

79. Vamathevan J, Clark D, Czodrowski P, Dunham I, Ferran E, Lee G, Li B, Madabhushi A, Shah P, Spitzer M, Zhao S (2019) Applications of machine learning in drug discovery and development. Nat Rev Drug Disc 18(6):463. https://doi.org/10.1038/s4157 3-019-0024-5

80. Brown N, Ertl P, Lewis R, Luksch T, Reker D, Schneider N (2020) Artificial intelligence in chemistry and drug design. J Comput Aided Mol Des 34(7):709. https://doi.org/10.1007/s1082 2-020-00317-x

81. Hertz JA, Krogh AS, Palmer RG (1991) Introduction to the theory of neural computation, 1st edn. Westview Press, Redwood City, CA

82. Hornik K, Stinchcombe M, White H (1989) Multilayer feedforward networks are universal approximators. Neural Netw 2(5):359. https://doi.org/10.1016/0893-6080(89)90020-8

83. Hornik K (1991) Approximation capabilities of multilayer feedforward networks. Neural Netw 4(2):251. https://doi org/10.1016/0893-6080(91)90009-T

84. Rasmussen CE, Williams CKI (2006) Gaussian processes for machine learning, vol 1. MIT Press, Cambridge

85. Behler J, Parrinello M (2007) Generalized neural-network representation of high-dimensional potential-energy surfaces. Phys Rev Lett 98(14):146401. https://doi.org/10.1103/PhysRevLet t.98.146401

86. Behler J (2011) Neural network potential-energy surfaces in chemistry: a tool for large-scale simulations. Phys Chem Chem Phys 13(40): 17930. https://doi.org/10.1039/C1CP21668F

87. Bartók AP, Payne MC, Kondor R, Csányi G (2010) Gaussian approximation potentials: the accuracy of quantum mechanics, without the electrons. Phys Rev Lett 104(13):136403. https:// doi.org/10.1103/PhysRevLett.104.136403

88. Bartók AP, Kondor R, Csányi G (2013) On representing chemical environments. Phys Rev B 87(18):184115. https://doi. org/10.1103/PhysRevB.87.184115

89. Botu V, Ramprasad R (2015) Adaptive machine learning framework to accelerate ab initio molecular dynamics. Int J Quantum Chem 115(16):1074. https://doi.org/10.1002/qua.24836

90. Blank TB, Brown SD, Calhoun AW, Doren DJ (1995) Neural network models of potential energy surfaces. J Chem Phys 103(10):4129. https://doi.org/10.1063/1.469597

91. Tafeit E, Estelberger W, Horejsi R, Moeller R, Oettl K, Vrecko K, Reibnegger G (1996) Neural networks as a tool for compact representation of ab initio molecular potential energy surfaces. J Mol Graph 14(1):12. https://doi.org/10.1016/02637855(95)00087-9

92. Brown DFR, Gibbs MN, Clary DC (1996) Combining ab initio computations, neural networks, and diffusion Monte Carlo: an efficient method to treat weakly bound molecules. J Chem Phys 105(17):7597. https://doi.org/10.1063/1.472596 
93. Tai No K, Ha Chang K, Yeon Kim S, Shik Jhon M, Scheraga HA (1997) Description of the potential energy surface of the water dimer with an artificial neural network, Chemical Physics Letters 271(1), 152. https://doi.org/10.1016/S0009-2614(97)00448-X

94. Lorenz S, Groß A, Scheffler M (2004) Representing highdimensional potential-energy surfaces for reactions at surfaces by neural networks. Chem Phys Lett 395(4):210. https://doi. org/10.1016/j.cplett.2004.07.076

95. Behler J (2011) Atom-centered symmetry functions for constructing high-dimensional neural network potentials. J Chem Phys 134(7):074106. https://doi.org/10.1063/1.3553717

96. Artrith N, Morawietz T, Behler J (2011) High-dimensional neural-network potentials for multicomponent systems: Applications to zinc oxide, Physical Review B 83(15). https://doi.org/10.1103/ PhysRevB.83.153101

97. Huang Y, Kang J, Goddard WA, Wang LW (2019) Density functional theory based neural network force fields from energy decompositions. Phys Rev B 99(6):064103. https://doi. org/10.1103/PhysRevB.99.064103

98. Nezbeda I, Kolafa J (1999) Effect of short- and long-range forces on the structure of water: temperature and density dependence. Mol Phys 97(10):1105. https://doi.org/10.1080/0026897990 9482911

99. Artrith N, Behler J (2012) High-dimensional neural network potentials for metal surfaces: A prototype study for copper, Physical Review B 85(4). https://doi.org/10.1103/PhysRevB.85.04543 9

100. Artrith N, Hiller B, Behler J (2013) Neural network potentials for metals and oxides-first applications to copper clusters at zinc oxide. Physica Status Solidi (B) 250(6):1191. https://doi. org/10.1002/pssb.201248370

101. Morawietz T (2015) Efficient simulations of water with ab initio accuracy: Development of high-dimensional neural network potentials for water clusters and bulk water. Ph.D. thesis, RuhrUniversity Bochum

102. Morawietz T, Sharma V, Behler J (2012) A neural network potential-energy surface for the water dimer based on environment-dependent atomic energies and charges. J Chem Phys 136(6):064103. https://doi.org/10.1063/1.3682557

103. Artrith N (2013) High-dimensional neural network potentials for solids and surfaces. Ph.D. thesis, Ruhr-University Bochum

104. Toukmaji AY, Board JA (1996) Ewald summation techniques in perspective: a survey. Comput Phys Commun 95(2):73. https:// doi.org/10.1016/0010-4655(96)00016-1

105. Fennell CJ, Gezelter JD (2006) Is the Ewald summation still necessary? Pairwise alternatives to the accepted standard for long-range electrostatics. J Chem Phys 124(23):234104. https:// doi.org/10.1063/1.2206581

106. Hirshfeld FL (1977) Bonded-atom fragments for describing molecular charge densities. Theor Chim Acta 44(2):129. https ://doi.org/10.1007/BF00549096

107. Wiberg KB, Rablen PR (1993) Comparison of atomic charges derived via different procedures. J Comput Chem 14(12):1504. https://doi.org/10.1002/jcc.540141213

108. Yao K, Herr J E, Toth D W, Mckintyre R, Parkhill J (2018) The TensorMol-0.1 model chemistry: a neural network augmented with long-range physics. Chemical Sci 9(8):2261. https://doi. org/10.1039/C7SC04934J

109. Deng Z, Chen C, Li XG, Ong SP (2019) An electrostatic spectral neighbor analysis potential for lithium nitride, npj Computational Materials 5(1), 1. https://doi.org/10.1038/s41524-019-0212-1

110. Grisafi A, Ceriotti M (2019) Incorporating long-range physics in atomic-scale machine learning. J Chem Phys 151(20):204105. https://doi.org/10.1063/1.5128375

111. Morawietz T, Behler J (2013) A density-functional theory-based neural network potential for water clusters including van der
Waals corrections. J Phys Chem A 117(32):7356. https://doi. org/10.1021/jp401225b

112. Grimme S, Antony J, Ehrlich S, Krieg H (2010) A consistent and accurate $\mathrm{ab}$ initio parametrization of density functional dispersion correction (DFT-D) for the 94 elements H-Pu. J Chem Phys 132(15):154104. https://doi.org/10.1063/1.3382344

113. Grimme S (2006) Semiempirical GGA-type density functional constructed with a long-range dispersion correction. J Comput Chem 27(15):1787. https://doi.org/10.1002/jcc.20495

114. Morawietz T, Singraber A, Dellago C, Behler J (2016) How van der Waals interactions determine the unique properties of water. Proc Nat Acad Sci 113(30):8368. https://doi.org/10.1073/ pnas. 1602375113

115. Xie T, Grossman JC (2018) Crystal Graph Convolutional Neural Networks for an Accurate and Interpretable Prediction of Material Properties, Physical Review Letters 120(14). https://doi. org/10.1103/PhysRevLett.120.145301

116. Chen C, Ye W, Zuo Y, Zheng C, Ong SP (2019) Graph networks as a universal machine learning framework for molecules and crystals. Chem Mater 31(9):3564. https://doi.org/10.1021/acs. chemmater.9b01294

117. Schütt KT, Kessel P, Gastegger M, Nicoli KA, Tkatchenko A, Müller KR (2019) SchNetPack: a deep learning toolbox for atomistic systems. J Chem Theory Comput 15(1):448. https:// doi.org/10.1021/acs.jctc.8b00908

118. Sadeghi A, Ghasemi SA, Schaefer B, Mohr S, Lill MA, Goedecker S (2013) Metrics for measuring distances in configuration spaces. J Chem Phys 139(18):184118. https://doi. org/10.1063/1.4828704

119. Schütt KT, Glawe H, Brockherde F, Sanna A, Müller KR, Gross EKU (2014) How to represent crystal structures for machine learning: towards fast prediction of electronic properties. Phys Rev B 89(20):205118. https://doi.org/10.1103/PhysR evB.89.205118

120. von Lilienfeld OA, Ramakrishnan R, Rupp M, Knoll A (2015) Fourier series of atomic radial distribution functions: a molecular fingerprint for machine learning models of quantum chemical properties. Int J Quantum Chem 115(16):1084. https://doi. org/10.1002/qua.24912

121. Faber F, Lindmaa A, von Lilienfeld OA, Armiento R (2015) Crystal structure representations for machine learning models of formation energies. Int J Quantum Chem 115(16):1094. https://doi.org/10.1002/qua.24917

122. Kearnes S, McCloskey K, Berndl M, Pande V, Riley P (2016) Molecular graph convolutions: moving beyond fingerprints. J Comput Aided Mol Des 30(8):595. https://doi.org/10.1007/ s10822-016-9938-8

123. Huo H, Rupp M (2018) Unified Representation of Molecules and Crystals for Machine Learning, arXiv:1704.06439 [condmat, physics:physics]

124. Zhang L, Han L, Wang H, Car R, W. E (2018) Deep Potential Molecular Dynamics: A Scalable Model with the Accuracy of Quantum Mechanics, Physical Review Letters 120(14), 143001. https://doi.org/10.1103/PhysRevLett.120.143001

125. Wang H, Zhang L, Han J, W. E (2018) DeePMD-kit: A deep learning package for many-body potential energy representation and molecular dynamics, Computer Physics Communications 228, 178. https://doi.org/10.1016/j.cpc.2018.03.016

126. Göller AH (2020) The art of atom descriptor design. Drug Discov Today Technol. https://doi.org/10.1016/j.ddtec .2020.06.004

127. Artrith N, Urban A (2016) An implementation of artificial neural-network potentials for atomistic materials simulations: performance for TiO2. Comput Mater Sci 114:135. https://doi. org/10.1016/j.commatsci.2015.11.047 
128. Khorshidi A, Peterson AA (2016) Amp: a modular approach to machine learning in atomistic simulations. Comput Phys Commun 207:310. https://doi.org/10.1016/j.cpc.2016.05.010

129. Smith JS, Isayev O, Roitberg AE (2017) ANI-1: an extensible neural network potential with DFT accuracy at force field computational cost. Chem Sci 8(4):3192. https://doi.org/10.1039/ C6SC05720A

130. Singraber A, Morawietz T, Behler J, Dellago C (2019) Parallel multistream training of high-dimensional neural network potentials. J Chem Theory Comput 15(5):3075. https://doi. org/10.1021/acs.jctc. 8 b01092

131. Ropo M, Schneider M, Baldauf C, Blum V (2016) First-principles data set of 45,892 isolated and cation-coordinated conformers of 20 proteinogenic amino acids. Sci Data 3(1):160009. https ://doi.org/10.1038/sdata.2016.9

132. Artrith N, Urban A, Ceder G (2017) Efficient and accurate machine-learning interpolation of atomic energies in compositions with many species, Physical Review B 96(1). https://doi. org/10.1103/PhysRevB.96.014112

133. Li L, Li H, Seymour ID, Koziol L, Henkelman G (2020) Pairdistribution-function guided optimization of fingerprints for atom-centered neural network potentials. J Chem Phys 152(22):224102. https://doi.org/10.1063/5.0007391

134. Gastegger M, Schwiedrzik L, Bittermann M, Berzsenyi F, Marquetand P (2018) wACSF-weighted atom-centered symmetry functions as descriptors in machine learning potentials. J Chem Phys 148(24):241709. https://doi.org/10.1063/1.5019667

135. Cooper AM, Kästner J, Urban A, Artrith N (2020) Efficient training of ANN potentials by including atomic forces via Taylor expansion and application to water and a transition-metal oxide, npj Computational Materials 6(1). https://doi.org/10.1038/s4152 4-020-0323-8

136. Chmiela S, Tkatchenko A, Sauceda HE, Poltavsky I, Schütt KT, Müller KR (2017) Machine learning of accurate energy-conserving molecular force fields. Sci Adv 3(5):e1603015. https://doi. org/10.1126/sciadv.1603015

137. Shen L, Yang W (2018) Molecular dynamics simulations with quantum mechanics/molecular mechanics and adaptive neural networks. J Chem Theory Comput 14(3):1442. https://doi. org/10.1021/acs.jctc.7b01195

138. Zhang P, Shen L, Yang W (2019) Solvation free energy calculations with quantum mechanics/molecular mechanics and machine learning models. J Phys Chem B 123(4):901. https:// doi.org/10.1021/acs.jpcb.8b11905

139. Hünenberger P, Reif M (2011) Single-ion solvation: experimental and theoretical approaches to elusive thermodynamic quantities. Royal Society of Chemistry, London

140. Jinnouchi R, Karsai F, Kresse G (2020) Making free-energy calculations routine: combining first principles with machine learning. Phys Rev B 101(6):060201. https://doi.org/10.1103/ PhysRevB.101.060201

141. Ruffo R, Hong SS, Chan CK, Huggins RA, Cui Y (2009) Impedance analysis of silicon nanowire lithium ion battery anodes. J Phys Chem C 113(26):11390. https://doi. org/10.1021/jp901594g

142. Ding N, Xu J, Yao YX, Wegner G, Fang X, Chen CH, Lieberwirth I (2009) Determination of the diffusion coefficient of lithium ions in nano-Si. Solid State Ionics 180(2):222. https://doi. org/10.1016/j.ssi.2008.12.015

143. Xie J, Imanishi N, Zhang T, Hirano A, Takeda Y, Yamamoto O (2010) Li-ion diffusion in amorphous Si films prepared by RF magnetron sputtering: a comparison of using liquid and polymer electrolytes. Mater Chem Phys 120(2):421. https://doi. org/10.1016/j.matchemphys.2009.11.031

144. Li J, Xiao X, Yang F, Verbrugge MW, Cheng YT (2012) Potentiostatic intermittent titration technique for electrodes governed by diffusion and interfacial reaction. J Phys Chem C 116(1):1472. https://doi.org/10.1021/jp207919q

145. Artrith N, Urban A, Wang Y, Ceder G (2019) Atomic-scale factors that control the rate capability of nanostructured amorphous Si for high-energy-density batteries, arXiv:1901.09272 [condmat, physics:physics]

146. Strauß F, Dörrer L, Bruns M, Schmidt H (2018) Lithium tracer diffusion in amorphous LixSi for low li concentrations. J Phys Chem C 122(12):6508. https://doi.org/10.1021/acs.jpcc.7b12296

147. Li W, Ando Y (2020) Effect of local structural disorder on lithium diffusion behavior in amorphous silicon, Physical Review Materials 4(4). https://doi.org/10.1103/PhysRevMaterial s. 4.045602

148. Kuwata N, Lu X, Miyazaki T, Iwai Y, Tanabe T, Kawamura J (2016) Lithium diffusion coefficient in amorphous lithium phosphate thin films measured by secondary ion mass spectroscopy with isotope exchange methods. Solid State Ionics 294:59. https ://doi.org/10.1016/j.ssi.2016.06.015

149. Li W, Ando Y, Minamitani E, Watanabe S (2017) Study of $\mathrm{Li}$ atom diffusion in amorphous Li3PO4 with neural network potential. J Chem Phys 147(21):214106. https://doi. org/10.1063/1.4997242

150. Kuhn A, Duppel V, Lotsch BV (2013) Tetragonal Li10GeP2S12 and Li7GePS8 - exploring the Li ion dynamics in LGPS Li electrolytes. Energy Environ Sci 6(12):3548. https://doi.org/10.1039/ C3EE41728J

151. Marcolongo A, Binninger T, Zipoli F, Laino T (2020) Simulating Diffusion Properties of Solid-State Electrolytes via a Neural Network Potential: Performance and Training Scheme, ChemSystemsChem 2(3). https://doi.org/10.1002/syst.201900031

152. Wang Y, Lai W (2015) Phase transition in lithium garnet oxide ionic conductors Li7La3Zr2O12: the role of Ta substitution and $\mathrm{H} 2 \mathrm{O} / \mathrm{CO} 2$ exposure. J Power Sources 275:612. https://doi. org/10.1016/j.jpowsour.2014.11.062

153. Wang C, Aoyagi K, Wisesa P, Mueller T (2020) Lithium ion conduction in cathode coating materials from on-the-fly machine learning. Chem Mater 32(9):3741. https://doi.org/10.1021/acs. chemmater.9b04663

154. Sorokin NI (2018) Characteristics of the Li+-Ion conductivity of Li3R2(PO4)3 crystals $(\mathrm{R}=\mathrm{Fe}, \mathrm{Sc})$ in the superionic state. Phys Solid State 60(5):899. https://doi.org/10.1134/S10637834180503 OX

155. Pilz T, Jansen M (2011) Li2B6O9F2, a New Acentric Fluorooxoborate. Zeitschrift für anorganische und allgemeine Chemie 637(14-15):2148. https://doi.org/10.1002/zaac.201100347

156. Court-Castagnet R (1993) Ionic conductivity-enhancement of $\mathrm{LiCl}$ by homogeneous and heterogeneous dopings. Solid State Ionics 61(4):327. https://doi.org/10.1016/0167-2738(93)90400 $-\mathrm{W}$

157. Cramer CJ, Truhlar DG (2001) Free energy calculations in rational drug design. Springer, Berlin

158. Elstner M, Porezag D, Jungnickel G, Elsner J, Haugk M, Frauenheim T, Suhai S, Seifert G (1998) Self-consistent-charge density-functional tight-binding method for simulations of complex materials properties. Phys Rev B 58(11):7260. https ://doi.org/10.1103/PhysRevB.58.7260

159. Ramakrishnan R, Dral PO, Rupp M, von Lilienfeld OA (2015) Big Data Meets Quantum Chemistry Approximations: The $\Delta$-Machine Learning Approach. J Chem Theory Comput 11(5):2087. https://doi.org/10.1021/acs.jctc.5b00099

160. Friedman JH (2001) Greedy function approximation: a gradient boosting machine. Ann Stat 29(5):1189

161. Jinnouchi R, Lahnsteiner J, Karsai F, Kresse G, Bokdam M (2019) Phase transitions of hybrid perovskites simulated by machine-learning force fields trained on the fly with 
bayesian inference. Phys Rev Lett 122(22):225701. https:// doi.org/10.1103/PhysRevLett.122.225701

162. Jinnouchi R, Karsai F, Kresse G (2019) On-the-fly machine learning force field generation: application to melting points. Phys Rev B 100(1):014105. https://doi.org/10.1103/PhysR evB.100.014105

163. Alongi KS, Shields GC (2010) in Annual Reports in Computational Chemistry, vol. 6, ed. by R.A. Wheeler (Elsevier), pp. 113-138. https://doi.org/10.1016/S1574-1400(10)06008-1

164. Zhang Y, Cremer PS (2006) Interactions between macromolecules and ions: the Hofmeister series. Curr Opin Chem Biol 10(6):658. https://doi.org/10.1016/j.cbpa.2006.09.020

165. Duignan TT, Baer MD, Schenter GK, Mundy CJ (2017) Real single ion solvation free energies with quantum mechanical simulation. Chem Sci 8(9):6131. https://doi.org/10.1039/ C7SC02138K

166. Zwanzig RW (1954) High-Temperature Equation of State by a Perturbation Method. I. Nonpolar Gases, The Journal of Chemical Physics 22(8), 1420. https://doi.org/10.1063/1.1740409

167. Kirkwood JG (1935) Statistical mechanics of fluid mixtures. J Chem Phys 3(5):300. https://doi.org/10.1063/1.1749657

168. Gastegger M, Behler J, Marquetand P (2017) Machine learning molecular dynamics for the simulation of infrared spectra. Chem Sci 8(10):6924. https://doi.org/10.1039/C7SC02267K

169. Vaden TD, de Boer TSJA, Simons JP, Snoek LC, Suhai S, Paizs B (2008) Vibrational spectroscopy and conformational structure of protonated polyalanine peptides isolated in the gas phase. J Phys Chem A 112(20):4608. https://doi.org/10.1021/ jp800069n

170. Morawietz T, Marsalek O, Pattenaude SR, Streacker LM, BenAmotz D, Markland TE (2018) The Interplay of Structure and Dynamics in the Raman Spectrum of Liquid Water over the Full Frequency and Temperature Range. The Journal of Physical Chemistry Letters 9(4):851. https://doi.org/10.1021/acs.jpcle tt. $8 \mathrm{~b} 00133$

171. Morawietz T, Urbina AS, Wise PK, Wu X, Lu W, Ben-Amotz D, Markland TE (2019) Hiding in the crowd: spectral signatures of overcoordinated hydrogen-bond environments. J Phys Chem Lett 10(20):6067. https://doi.org/10.1021/acs.jpclett.9b01781

172. Raimbault N, Grisafi A, Ceriotti M, Rossi M (2019) Using Gaussian process regression to simulate the vibrational Raman spectra of molecular crystals. New J Phys 21(10):105001. https ://doi.org/10.1088/1367-2630/ab4509

173. Datta S, Grant DJW (2004) Crystal structures of drugs: Advances in determination, prediction and engineering. Nat Rev Drug Discovery 3(1):42. https://doi.org/10.1038/nrd1280

174. Gastegger M, Marquetand P (2015) High-dimensional neural network potentials for organic reactions and an improved training algorithm. J Chem Theory Comput 11(5):2187. https://doi. org/10.1021/acs.jctc.5b00211

175. Kato K, Masuda T, Watanabe C, Miyagawa N, Mizouchi H, Nagase S, Kamisaka K, Oshima K, Ono S, Ueda H, Tokuhisa A, Kanada R, Ohta M, Ikeguchi M, Okuno Y, Fukuzawa K, Honma T (2020) High-precision atomic charge prediction for protein systems using fragment molecular orbital calculation and machine learning. J Chem Inf Model. https://doi.org/10.1021/acs. jcim.0c00273

176. Shang H, Raimbault N, Rinke P, Scheffler M, Rossi M, Carbogno C (2018) All-electron, real-space perturbation theory for homogeneous electric fields: theory, implementation, and application within DFT. New J Phys 20(7):073040. https://doi. org/10.1088/1367-2630/aace6d

177. Raimbault N, Athavale V, Rossi M (2019) Anharmonic effects in the low-frequency vibrational modes of aspirin and paracetamol crystals. Phys Rev Mater 3(5):053605. https://doi.org/10.1103/ PhysRevMaterials.3.053605

178. Grisafi A, Wilkins DM, Csányi G, Ceriotti M (2018) Symmetryadapted machine learning for tensorial properties of atomistic systems. Phys Rev Lett 120(3):036002. https://doi.org/10.1103/ PhysRevLett.120.036002

179. Kapil V, Wilkins DM, Lan J, Ceriotti M (2020) Inexpensive modeling of quantum dynamics using path integral generalized Langevin equation thermostats. J Chem Phys 152(12):124104. https://doi.org/10.1063/1.5141950

180. Paruzzo FM, Hofstetter A, Musil F, De S, Ceriotti M, Emsley L (2018) Chemical shifts in molecular solids by machine learning. Nature Commun 9(1):4501. https://doi.org/10.1038/s41467-01806972-x

181. Piana S, Sebastiani D, Carloni P, Parrinello M (2001) Ab initio molecular dynamics-based assignment of the protonation state of pepstatin A/HIV-1 protease cleavage site. J Am Chem Soc 123(36):8730. https://doi.org/10.1021/ja003145e

182. de Dios A, Pearson JG, Oldfield E (1993) Secondary and tertiary structural effects on protein NMR chemical shifts: an ab initio approach. Science 260(5113):1491. https://doi.org/10.1126/scien ce. 8502992

183. Sebastiani D, Parrinello M (2001) A new ab-initio approach for NMR chemical shifts in periodic systems. J Phys Chem A 105(10):1951. https://doi.org/10.1021/jp002807j

184. Sumowski CV, Hanni M, Schweizer S, Ochsenfeld C (2014) Sensitivity of ab initio vs empirical methods in computing structural effects on NMR chemical shifts for the example of peptides. J Chem Theory Comput 10(1):122. https://doi.org/10.1021/ct400 $713 \mathrm{t}$

185. Mifsud N, Elena B, Pickard CJ, Lesage A, Emsley L (2006) Assigning powders to crystal structures by high-resolution $1 \mathrm{H}-1 \mathrm{H}$ double quantum and $1 \mathrm{H}-13 \mathrm{C}$ J-INEPT solid-state NMR spectroscopy and first principles computation. A case study of penicillin G, Physical Chemistry Chemical Physics 8(29):3418. https://doi.org/10.1039/B605227D

186. Baias M, Dumez JN, Svensson PH, Schantz S, Day GM, Emsley L (2013) De Novo determination of the crystal structure of a large drug molecule by crystal structure prediction-based powder NMR crystallography. J Am Chem Soc 135(46):17501. https:// doi.org/10.1021/ja4088874

187. Groom CR, Bruno IJ, Lightfoot MP, Ward SC (2016) The Cambridge structural database. Acta Crystallogr Section B 72(2):171. https://doi.org/10.1107/S2052520616003954

188. Musil F, Willatt MJ, Langovoy MA, Ceriotti M (2019) Fast and accurate uncertainty estimation in chemical machine learning. J Chem Theory Comput 15(2):906. https://doi.org/10.1021/acs. jctc.8b00959

189. http://shiftml.epfl.ch (2019)

190. Artrith N (2019) Machine learning for the modeling of interfaces in energy storage and conversion materials. J Phys Energy 1(3):032002. https://doi.org/10.1088/2515-7655/ab2060

191. Aykol M, Dwaraknath SS, Sun W, Persson KA (2018) Thermodynamic limit for synthesis of metastable inorganic materials, Science Advances 4(4), eaaq0148. https://doi.org/10.1126/sciad v.aaq0148

192. Robinson VN, Zong H, Ackland GJ, Woolman G, Hermann A (2019) On the chain-melted phase of matter. Proc Nat Acad Sci 116(21): 10297

193. Niu H, Bonati L, Piaggi PM, Parrinello M (2020) Ab initio phase diagram and nucleation of gallium. Nature Commun 11(1):2654. https://doi.org/10.1038/s41467-020-16372-9

194. Andolina CM, Williamson P, Saidi WA (2020) Optimization and validation of a deep learning $\mathrm{CuZr}$ atomistic potential: robust applications for crystalline and amorphous phases with 
near-DFT accuracy. J Chem Phys 152(15):154701. https://doi. org/10.1063/5.0005347

195. Sosso GC, Miceli G, Caravati S, Giberti F, Behler J, Bernasconi M (2013) Fast crystallization of the phase change compound GeTe by large-scale molecular dynamics simulations. J Phys Chem Lett 4(24):4241. https://doi.org/10.1021/jz402268v

196. Gabardi S, Sosso GG, Behler J, Bernasconi M (2019) Priming effects in the crystallization of the phase change compound GeTe from atomistic simulations. Faraday Discuss 213:287. https://doi. org/10.1039/C8FD00101D

197. Barducci A, Bussi G, Parrinello M (2008) Well-tempered metadynamics: a smoothly converging and tunable free-energy method. Phys Rev Lett 100(2):020603. https://doi.org/10.1103/ PhysRevLett.100.020603

198. Behler J, Martoňák R, Donadio D, Parrinello M (2008) Metadynamics simulations of the high-pressure phases of silicon employing a high-dimensional neural network potential. Phys Rev Lett 100(18):185501. https://doi.org/10.1103/PhysRevLet t.100.185501

199. Eshet H, Khaliullin RZ, Kühne TD, Behler J, Parrinello M (2010) $\mathrm{Ab}$ initio quality neural-network potential for sodium. Phys Rev B 81(18):184107. https://doi.org/10.1103/PhysRevB.81.184107

200. Bonati L, Parrinello M (2018) Silicon liquid structure and crystal nucleation from Ab initio deep metadynamics. Phys Rev Lett 121(26):265701. https://doi.org/10.1103/PhysRevLett.121.26570 1

201. Kong FC, Li YF, Shang C, Liu ZP (2019) Stability and phase transition of cobalt oxide phases by machine learning global potential energy surface. J Phys Chem C 123(28):17539. https:// doi.org/10.1021/acs.jpcc.9b02842

202. Artrith N, Kolpak AM (2014) Understanding the composition and activity of electrocatalytic nanoalloys in aqueous solvents: a combination of DFT and accurate neural network potentials. Nano Lett 14(5):2670. https://doi.org/10.1021/n15005674

203. Artrith N, Kolpak AM (2015) Grand canonical molecular dynamics simulations of $\mathrm{Cu}$-Au nanoalloys in thermal equilibrium using reactive ANN potentials. Comput Mater Sci 110:20. https://doi. org/10.1016/j.commatsci.2015.07.046

204. Artrith N, Urban A, Ceder G (2018) Constructing first-principles phase diagrams of amorphous $\mathrm{Li}_{x} \mathrm{Si}$ using machine-learningassisted sampling with an evolutionary algorithm. J Chem Phys 148(24):241711. https://doi.org/10.1063/1.5017661

205. Sun G, Sautet P (2018) Metastable structures in cluster catalysis from first-principles: structural ensemble in reaction conditions and metastability triggered reactivity. J Am Chem Soc 140(8):2812. https://doi.org/10.1021/jacs.7b11239

206. Schmal M (2016) Heterogeneous catalysis and its industrial applications, 1st edn. Springer, New York, NY

207. Medford AJ, Vojvodic A, Hummelshøj JS, Voss J, Abild-Pedersen F, Studt F, Bligaard T, Nilsson A, Nørskov JK (2015) From the Sabatier principle to a predictive theory of transition-metal heterogeneous catalysis. J Catal 328:36. https://doi.org/10.1016/j. jcat.2014.12.033

208. Goldsmith BR, Esterhuizen J, Liu JX, Bartel CJ, Sutton C (2018) Machine learning for heterogeneous catalyst design and discovery. AIChE J 64(7):2311. https://doi.org/10.1002/aic.16198

209. Kitchin JR (2018) Machine learning in catalysis. Nature Catalysis 1(4):230. https://doi.org/10.1038/s41929-018-0056-y

210. Hansen PL, Wagner JB, Helveg S, Rostrup-Nielsen JR, Clausen BS, Topsøe H (2002) Atom-resolved imaging of dynamic shape changes in supported copper nanocrystals. Science 295(5562):2053. https://doi.org/10.1126/science.1069325
211. Behrens M, Studt F, Kasatkin I, Kühl S, Hävecker M, AbildPedersen F, Zander S, Girgsdies F, Kurr P, Kniep BL, Tovar M, Fischer RW, Nørskov JK, Schlögl R (2012) The active site of methanol synthesis over $\mathrm{Cu} / \mathrm{ZnO} / \mathrm{Al} 2 \mathrm{O} 3$ industrial catalysts. Science 336(6083):893. https://doi.org/10.1126/science.1219831

212. Kolsbjerg EL, Peterson AA, Hammer B (2018) Neural-networkenhanced evolutionary algorithm applied to supported metal nanoparticles. Phys Rev B 97(19):195424. https://doi.org/10.1103/ PhysRevB.97.195424

213. Onat B, Cubuk ED, Malone BD, Kaxiras E (2018) Implanted neural network potentials: application to Li-Si alloys. Phys Rev B 97(9):094106. https://doi.org/10.1103/PhysRevB.97.094106

214. Lacivita V, Artrith N, Ceder G (2018) Structural and compositional factors that control the li-ion conductivity in LiPON electrolytes. Chem Mater 30(20):7077. https://doi.org/10.1021/acs. chemmater.8b02812

215. McDowell MT, Lee SW, Nix WD, Cui Y (2013) 25th anniversary article: understanding the lithiation of silicon and other alloying anodes for lithium-ion batteries. Adv Mater 25(36):4966. https ://doi.org/10.1002/adma.201301795

216. Chen MS, Morawietz T, Markland TE, Artrith N (2020) AENETLAMMPS and AENET-TINKER: Interfaces for accurate and efficient molecular dynamics simulations with machine learning potentials, Materials Cloud Archive 2020.92. https://doi. org/10.24435/materialscloud:dx-ct

217. Takada K (2013) Progress and prospective of solid-state lithium batteries. Acta Mater 61(3):759. https://doi.org/10.1016/j.actam at.2012.10.034

218. Famprikis T, Canepa P, Dawson JA, Islam MS, Masquelier C (2019) Fundamentals of inorganic solid-state electrolytes for batteries. Nat Mater 18(12):1278. https://doi.org/10.1038/s4156 3-019-0431-3

219. Bates JB, Dudney NJ, Gruzalski GR, Zuhr RA, Choudhury A, Luck CF, Robertson JD (1992) Electrical properties of amorphous lithium electrolyte thin films. Solid State Ionics 53-56:647. https://doi.org/10.1016/0167-2738(92)90442-R

220. Li W, Ando Y, Watanabe S (2017) Cu diffusion in amorphous $\mathrm{Ta}_{2} \mathrm{O}_{5}$ Studied with a simplified neural network potential. J Phys Soc Jpn 86(10):104004. https://doi.org/10.7566/JPSJ.86.104004

221. Zhang Y, He X, Chen Z, Bai Q, Nolan AM, Roberts CA, Banerjee D, Matsunaga T, Mo Y, Ling C (2019) Unsupervised discovery of solid-state lithium ion conductors, Nature Communications 10(1). https://doi.org/10.1038/s41467-019-13214-1

222. Cubuk ED, Sendek AD, Reed EJ (2019) Screening billions of candidates for solid lithium-ion conductors: a transfer learning approach for small data. J Chem Phys 150(21):214701. https:// doi.org/10.1063/1.5093220

223. Wang Z, Han Y, Li J, He X (2020) Combining the Fragmentation Approach and Neural Network Potential Energy Surfaces of Fragments for Accurate Calculation of Protein Energy. J Phys Chem B 124(15):3027. https://doi.org/10.1021/acs.jpcb.0c01370

224. Smith JS, Nebgen BT, Zubatyuk R, Lubbers N, Devereux C, Barros K, Tretiak S, Isayev O, Roitberg AE (2019) Approaching coupled cluster accuracy with a general-purpose neural network potential through transfer learning. Nature Commun 10(1):2903. https://doi.org/10.1038/s41467-019-10827-4

225. Riniker S (2017) Molecular dynamics fingerprints (MDFP): machine learning from MD data to predict free-energy differences. J Chem Inf Model 57(4):726. https://doi.org/10.1021/acs. jcim.6b00778 